\title{
Retrogressive failure of a static granular layer on an inclined plane
}

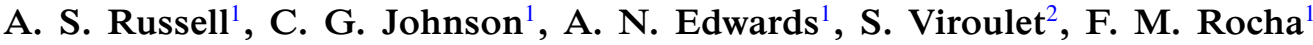 \\ and J. M. N. T. Gray ${ }^{1, \dagger}$ \\ ${ }^{1}$ School of Mathematics and Manchester Centre for Nonlinear Dynamics, University of Manchester, \\ Manchester M13 9PL, UK \\ ${ }^{2}$ Institut de Mécanique des Fluides de Toulouse (IMFT) - Université de Toulouse, CNRS, \\ 31400 Toulouse, France
}

(Received 23 November 2018; revised 4 March 2019; accepted 12 March 2019; first published online 26 April 2019)

When a layer of static grains on a sufficiently steep slope is disturbed, an upslope-propagating erosion wave, or retrogressive failure, may form that separates the initially static material from a downslope region of flowing grains. This paper shows that a relatively simple depth-averaged avalanche model with frictional hysteresis is sufficient to capture a planar retrogressive failure that is independent of the cross-slope coordinate. The hysteresis is modelled with a non-monotonic effective basal friction law that has static, intermediate (velocity decreasing) and dynamic (velocity increasing) regimes. Both experiments and time-dependent numerical simulations show that steadily travelling retrogressive waves rapidly form in this system and a travelling wave ansatz is therefore used to derive a one-dimensional depth-averaged exact solution. The speed of the wave is determined by a critical point in the ordinary differential equation for the thickness. The critical point lies in the intermediate frictional regime, at the point where the friction exactly balances the downslope component of gravity. The retrogressive wave is therefore a sensitive test of the functional form of the friction law in this regime, where steady uniform flows are unstable and so cannot be used to determine the friction law directly. Upper and lower bounds for the existence of retrogressive waves in terms of the initial layer depth and the slope inclination are found and shown to be in good agreement with the experimentally determined phase diagram. For the friction law proposed by Edwards et al. (J. Fluid. Mech., vol. 823, 2017, pp. 278-315, J. Fluid. Mech., 2019, (submitted)) the magnitude of the wave speed is slightly under-predicted, but, for a given initial layer thickness, the exact solution accurately predicts an increase in the wave speed with higher inclinations. The model also captures the finite wave speed at the onset of retrogressive failure observed in experiments.

Key words: geophysical and geological flows, granular media, waves/free-surface flows

$\dagger$ Email address for correspondence: nico.gray@manchester.ac.uk 


\section{Introduction}

Snow avalanches, debris flows, pyroclastic flows and submarine landslides often occur on inclines covered by a static layer of erodible granular material. A small disturbance to this layer, due either to human activity or natural processes such as additional snowfall, rainfall infiltration or earthquakes, may destabilise a small region and cause it to flow downslope. The presence of erodible material downslope of the disturbance allows the flow to grow rapidly in size as it erodes additional material (Mangeney et al. 2010; Iverson \& Ouyang 2015; Köhler et al. 2016). Sometimes static material is also eroded upslope of the disturbance through an upward-propagating wave of material failure. This 'retrogressive failure' (Varnes 1978) further increases the volume of the flow and is commonly observed in landslides as well as sand avalanches on the slip face of dunes. Submarine retrogressive failures are important in dredging processes, occurring when sediment is removed from the bottom or middle of a slope (Van den Berg, Van Gelder \& Mastbergen 2002; Eke, Viparelli \& Parker 2011; Mastbergen et al. 2016), but also occur naturally at continental margins and can generate substantial tsunamis (Lovholt et al. 2015; Glimsdal et al. 2016).

Retrogressive failures are also observed in small-scale dry granular flow experiments (e.g. Daerr \& Douady 1999; Daerr 2001, and those performed in this paper). Figure 1 shows an example of an approximately planar retrogressive wavefront, generated by a straight perturbation across the full width of the inclined plane (figure 2). The retrogressive wave separates a thicker upslope layer of static grains from a thinner downstream layer of flowing grains. In the experiments of Daerr \& Douady (1999) and Daerr (2001) the failure was initiated at a single point and so generated a non-planar retrogressive failure, instead. In their experiments a static layer of thickness $h_{\text {stop }}(\zeta)$ was created by pouring 180-300 $\mu \mathrm{m}$ diameter glass beads down a plane covered with velvet cloth and inclined at an angle $\zeta$ to the horizontal. Due to frictional hysteresis, the layer remained static until the inclination angle was increased, up to an angle $\zeta_{\text {start }}(h)>\zeta$, at which the layer spontaneously started flowing. Inclining the static layer to an angle only slightly larger than $\zeta$ and then perturbing it, resulted in an avalanche that propagated only downhill from the point of disturbance, whereas perturbing a layer that had been inclined further caused an additional upward retrogressive failure, propagating at a constant speed. Daerr (2001) showed that, at a certain inclination angle, the behaviour immediately switched from a downslope avalanche to a retrogressive failure in which the wave propagated upslope at a non-zero speed, i.e. there is a finite wave speed at the onset of retrogressive failure. The same qualitative behaviour also occurs in the retrogressive failures studied in this paper, but the fact that they are planar makes them more amenable to analysis.

Daerr \& Douady (1999) and Daerr (2001) termed the retrogressive failures 'uphill-propagating avalanches'. These were investigated theoretically by Bouchaud \& Cates (1998) using the Bouchaud, Cates, Ravi Prakash and Edwards (BCRE) model (Bouchaud et al. 1994) in which a granular material is separated into two phases; rolling and static. Although this was a purely kinematic model, it was able to capture the transition between non-retrogressive and retrogressive failure. However, with the BCRE model this transition occurred smoothly, i.e. there was a smooth variation of the retrogressive wave speed starting from zero when the wave does not move upslope, which contradicts the experimental results of Daerr (2001). Aranson \& Tsimring $(2001,2002)$ captured the retrogressive failures using a partially fluidised granular flow model with an order parameter equation to represent the transition between static and flowing grains. Using this model they found that 


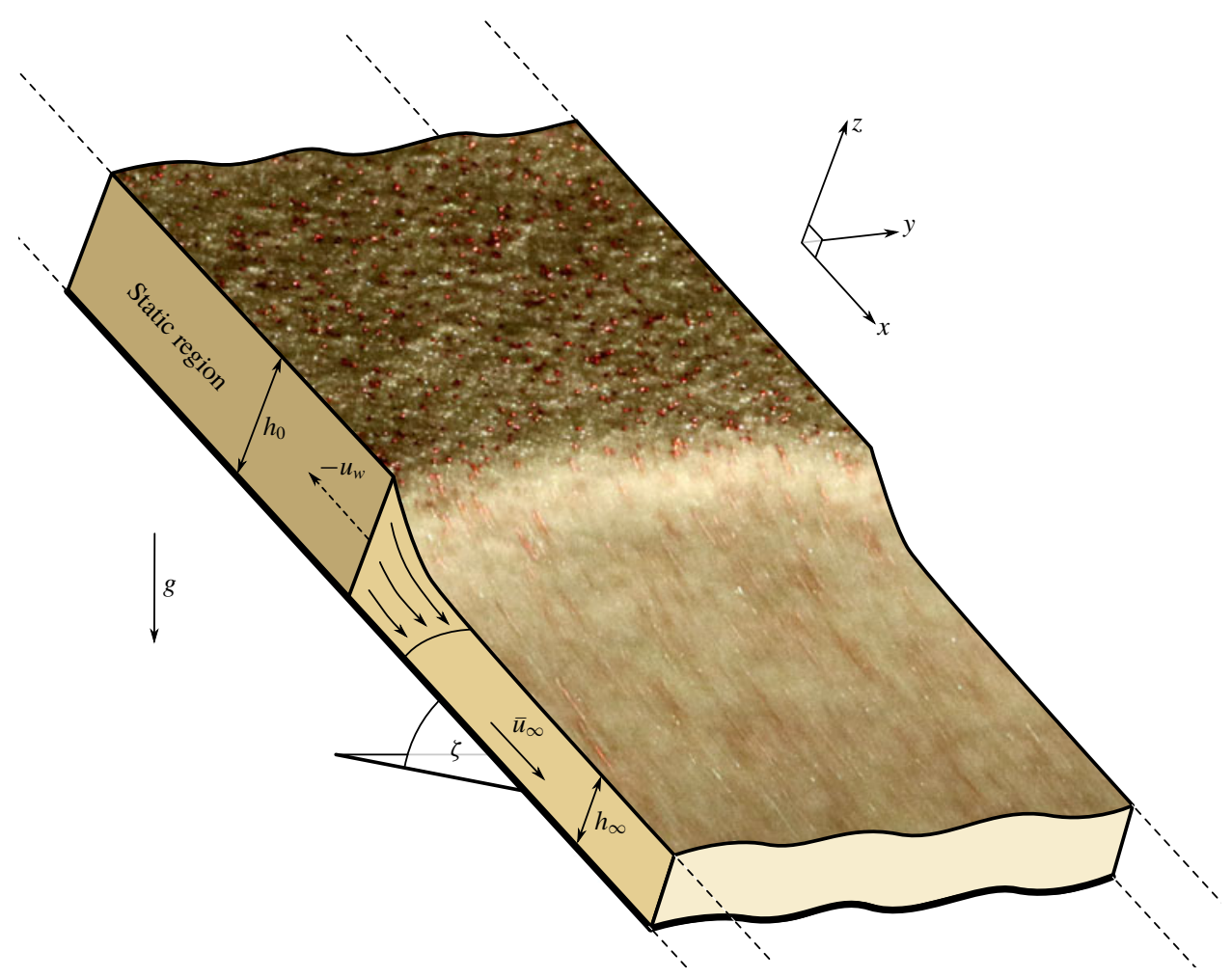

FIGURE 1. A composite of a sketch and a photograph of a planar retrogressive failure (approximately uniform in the cross-slope direction) in a small-scale laboratory experiment on a plane inclined at $\zeta$ to the horizontal. Static glass beads in a layer of thickness $h_{0} \in\left[h_{\text {stop }}(\zeta), h_{\text {start }}(\zeta)\right]$ (upper half of the photograph) are separated from a thinner flow of grains (lower half, blurred by the exposure time of $1 / 20 \mathrm{~s}$ ), of thickness $h_{\infty}$ and depth-averaged downslope velocity $\bar{u}_{\infty}$, by the front which propagates upslope with constant speed $u_{w}<0$ and progressively erodes the static layer. The transparent glass beads (125-160 $\mu \mathrm{m}$ diameter, appearing white) are seeded with red tracer particles (300-400 $\mu \mathrm{m}$ diameter) to aid visualisation. The time-dependent evolution of the wave can be seen in supplementary movies 1 and 2, which are available in the online supplementary material (https://doi.org/10.1017/jfm.2019.215).

the uphill-propagating front was a travelling wave solution with the onset occurring through a discontinuity in the speed of the retrogressive waves, i.e. the wave speed immediately jumped up from zero (for no upslope propagation) to a finite non-zero value. Their theoretical prediction for the existence of uphill waves agreed with the experimental data of Daerr \& Douady (1999) and Daerr (2001), using one fitting parameter. These models of retrogressive failure (Aranson \& Tsimring 2001, 2002) are, however, phenomenological in nature (Aradian, Raphaël \& De Gennes 2002), since the grain inertia is neglected and there is some uncertainty in the physics underlying the order parameter and its governing equation.

In this paper, it is shown that the retrogressive wave speed, as well as the jump in the wave speed at the non-retrogressive/retrogressive failure transition, can be quantitatively predicted by using a depth-averaged avalanche model with a non-monotonic effective basal friction law (Pouliquen \& Forterre 2002; Edwards 


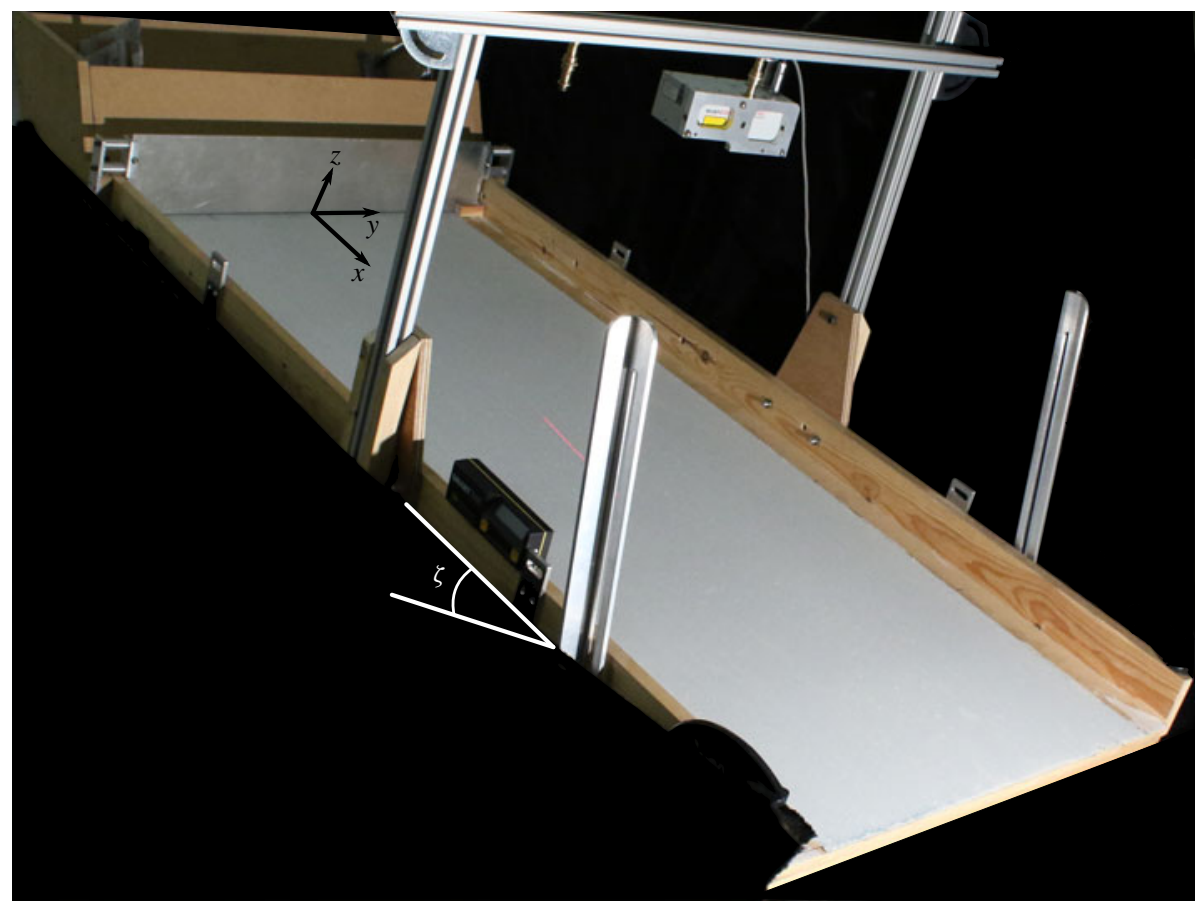

FIGURE 2. The experimental set-up consisting of a rough plane, inclined at an angle $\zeta$ to the horizontal, which has a monolayer of spherical glass beads of diameter 750-1000 $\mu \mathrm{m}$ stuck to the surface, to create no slip at the base. A hopper and a gate at the top of the chute are used to create a deposit of thickness $h_{\text {stop }}$ by generating a steady uniform flow and then closing the mass supply. The laser profilometer (Micro-Epsilon scanCONTROL 2700-100) is mounted normal to the inclined plane in order to measure the flow thickness. A set of coordinates $O x y z$ is defined with the $x$-axis pointing downslope, the $y$-axis across the slope and the $z$-axis along the upward pointing normal. A typical retrogressive wave experiment is shown in movie 3 in the online supplementary material.

et al. 2017, 2019). The non-monotonic friction law encodes the hysteresis that leads to $h_{\text {stop }}(\zeta)$ and $h_{\text {start }}(\zeta)$ (the inverse function of $\zeta_{\text {start }}(h)$ ) and consists of (i) a dynamic regime, which is a monotonically increasing function of the speed at moderate to high Froude numbers (Pouliquen 1999a), (ii) a multi-valued static friction when the Froude number is zero and (iii) a monotonically decreasing regime that interpolates between the maximum static friction and the minimum dynamic friction at low Froude numbers (Pouliquen \& Forterre 2002). In addition, Edwards et al. (2017, 2019) showed that there was another important thickness $h_{*} \in\left(h_{\text {stop }}, h_{\text {start }}\right)$, which defines the transition between intermediate and dynamic regimes and is the minimum observable steady uniform flow thickness.

Depth-integrated avalanche theories of this sort are widely used for modelling shallow granular free-surface flows and are closely related to the shallow water, or St Venant equations, of fluid mechanics. The first derivation for granular flows was by Savage \& Hutter $(1989,1991)$ assuming an incompressible flow with a Mohr-Coulomb rheology and a constant Coulomb basal friction coefficient. This yielded a shallow-water-like system of conservation laws with additional source terms due to gravity, basal friction and topography gradients, as well as an 'earth 
pressure' coefficient multiplying the depth-integrated pressure, which took active and passive values dependent on whether the flows were dilatational or compressional. In this paper, as in many recent works, the earth pressure coefficient is assumed to be unity (Gray, Wieland \& Hutter 1999; Pouliquen 1999b; Gray, Tai \& Noelle 2003). The model has been generalised to two-dimensional flows over complex topography (Gray et al. 1999; Wieland, Gray \& Hutter 1999; Mangeney-Castelnau et al. 2003; Pudasaini \& Hutter 2003; Bouchut \& Westdickenberg 2004; Luca et al. 2009) and has been widely used in the snow avalanche community for hazard zone mapping (Grigorian, Eglit \& Iakimov 1967; Naaim et al. 2004; Sampl \& Zwinger 2004; Christen, Kowalski \& Bartelt 2010; Fischer, Kowalski \& Pudasaini 2012). Analogous theories have been developed for debris flows (Iverson 1997; Iverson \& Denlinger 2001; Denlinger \& Iverson 2001; Johnson et al. 2012), pyroclastic flows (Pitman et al. 2003; Mangeney et al. 2007; Doyle, Hogg \& Mader 2011) and landslides (Kuo et al. 2009; Mangeney et al. 2010).

The constant Coulomb basal friction of Savage \& Hutter (1989) provides a good description of dry granular flows over smooth surfaces. However, the basal friction felt by flows over a rough surface, i.e. where the bed roughness approaches or exceeds the mean particle diameter, is strongly dependent on both the flow thickness and the speed (Pouliquen \& Forterre 2002). Gray \& Edwards (2014) showed that by formally depth averaging the $\mu(I)$-rheology for granular flows (GDR-MiDi 2004; Jop, Forterre \& Pouliquen 2006) and exploiting the shallowness of the system, the shallow-waterlike avalanche equations emerge naturally, at leading order in the aspect ratio, with an effective basal friction corresponding to Pouliquen \& Forterre's (2002) dynamic regime described above. However, since frictional hysteresis is key to the formation of retrogressive failures, it is necessary to augment the model with the intermediate and static regimes, at low and zero Froude numbers respectively (Pouliquen \& Forterre 2002; Edwards et al. 2017, 2019).

The retrogressive failures are particularly sensitive to the intermediate (velocitydecreasing) part of the friction law, which, in the absence of direct experimental observations, has previously been described by a power-law interpolation between the maximum static and minimum dynamic friction (Pouliquen \& Forterre 2002). Retrogressive failures are therefore not just of fundamental scientific interest, but also provide an important test case for the friction law proposed by Edwards et al. (2017, 2019).

\section{Experimental observations}

The experimental set-up consists of a plane ( $1.65 \mathrm{~m}$ long by $0.58 \mathrm{~m}$ wide) that is inclined at an angle $\zeta$ to the horizontal as shown in figure 2 . The plane is roughened with a monolayer of spherical glass beads of diameter 750-1000 $\mu \mathrm{m}$ (to ensure no basal slip, Silbert et al. 2001; Goujon, Thomas \& Dalloz-Dubrujeaud 2003; Jing et al. 2016), which are attached using double-sided sticky tape. To start an experiment, the plane is inclined to an angle $\zeta_{0}$ and glass beads, of diameter $125-160 \mu \mathrm{m}$, are released from a hopper at the top of the chute to form a steady uniform flow. As this flow ceases, it forms a static layer of uniform thickness $h_{0}=h_{\text {stop }}\left(\zeta_{0}\right)$ at its trailing edge (Edwards et al. 2019). The inclination of the chute is then carefully increased to an angle $\zeta>\zeta_{0}$. Provided that the new inclination angle is not too large, i.e. $\zeta<\zeta_{\text {start }}\left(h_{0}\right)$, frictional hysteresis keeps the grains static. The resulting layer is described as being metastable (Daerr 2001) because it can exist in either a flowing or static state, where the static state is stable to infinitesimal perturbations, but unstable to sufficiently large finite perturbations. 


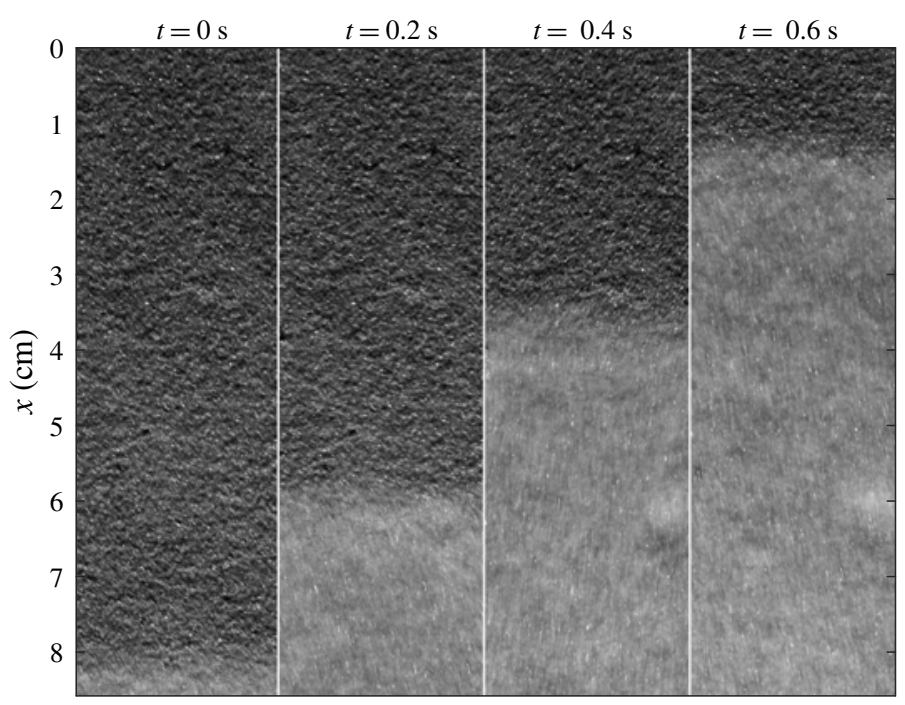

FIGURE 3. Overhead photographs showing a time sequence of the retrogressive failure front. It spans the width of each image and separates the initially stationary layer of 125$160 \mu \mathrm{m}$ glass beads (in focus at the top of each picture) from the flowing material below (blurred by the exposure time of $1 / 50 \mathrm{~s}$ ). The retrogressive front propagates upslope with speed $\left|u_{w}\right|=11.7 \mathrm{~cm} \mathrm{~s}^{-1}$, and continues to do so until it reaches the top of the static layer. The time-dependent evolution of the wave can be seen in movie 4 in the online supplementary material.

The layer is destabilised by tapping it near the bottom with a ruler held horizontally across the chute. After a rapid initial transient, the retrogressive failure propagates upwards at a constant speed, and remains approximately straight and perpendicular to the downslope direction as shown in figure 3 and supplementary movies $1-4$. The grains that have been eroded by this retrogressive failure form a steady uniform flow of thickness $h_{\infty}<h_{0}$ downstream of the wavefront. The propagation speed of the retrogressive front is determined by a high-speed camera (Teledyne DALSA Genie HM1400), which takes overhead photographs at 200 frames per second. Figure 4 shows a typical space-time plot that is constructed by extracting the middle row of pixels down the centre of the chute from each image and combining them into a single plot with time $t$ on the abscissa and downstream distance $x$ on the ordinate. Oblique illumination from the bottom of the chute makes the retrogressive front clearly visible as a diagonal line. The gradient of this line is measured to obtain the front propagation speed $\left|u_{w}\right|$.

Measurements of the flow thickness using a laser profilometer (Micro-Epsilon scanCONTROL 2700-100) are used to quantify the decrease in flow thickness $h$ that occurs across the retrogressive failure. For a typical experimental flow, illustrated in figure $5(a)$, this decrease is $\sim 0.13 \mathrm{~mm}$, which is approximately one grain diameter and is similar to the measured roughness of the free-surface profile. An average of many such thickness profiles in a frame moving upslope with the wave, at speed $\left|u_{w}\right|$, and plotted with respect to the frame coordinate $\xi=x-u_{w} t$ (figure $5 b$ ) provides a clearer picture of the shape of the retrogressive wave. Upslope of the wave $(\xi<0)$ the grains form a uniform thickness static layer. At the point of failure $\xi=0$ the surface gradient $\partial h / \partial \xi$ rapidly steepens and the flow transitions smoothly to a 


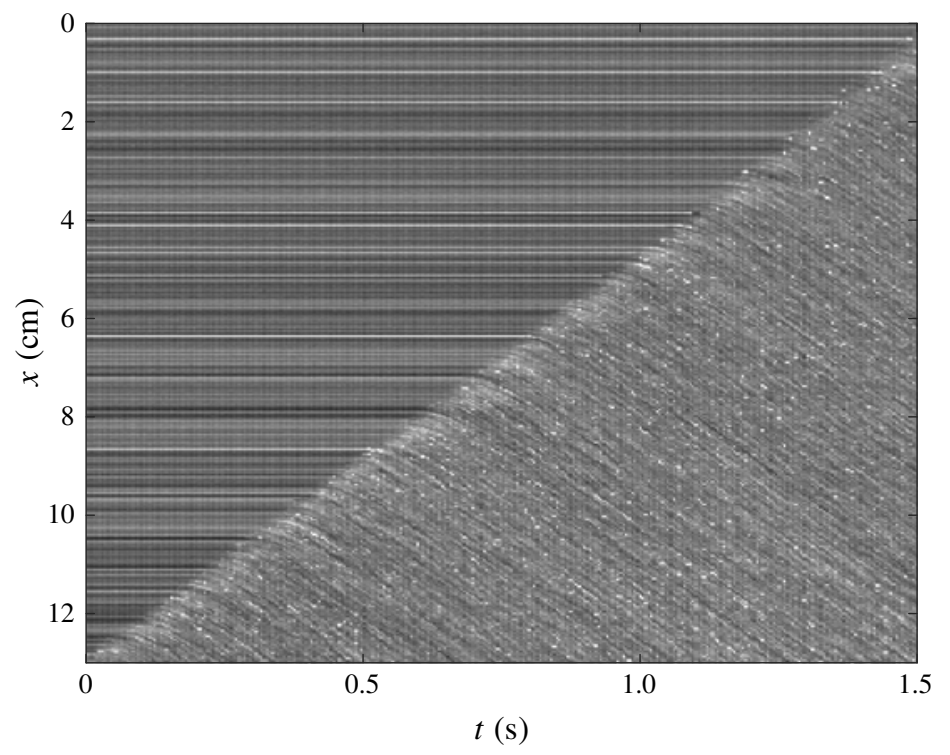

FIGURE 4. A space-time plot for $\zeta_{0}=24^{\circ}, \zeta=27.5^{\circ}$ and $h_{0}=h_{\text {stop }}\left(24^{\circ}\right)=0.95 \mathrm{~mm}$. The horizontal straight lines indicate material that is static and the more speckled region indicates flowing material. The interface between these two regions is the retrogressive failure front, which propagates upslope at speed $\left|u_{w}\right|=8.5 \mathrm{~cm} \mathrm{~s}^{-1}$. The steady uniform flow downslope of the front causes faint diagonal lines whose gradient implies that the surface velocity rapidly accelerates to $u_{s}=8.1 \mathrm{~cm} \mathrm{~s}^{-1}$.

thinner, uniform flowing layer downslope of the failure. The averaged experimental data in figure $5(b)$ indicate that this transition occurs over a length scale of 6-7 mm, although instantaneous measurements (figure $5 a$ ) suggest that the wave may be slightly narrower $(<5 \mathrm{~mm})$. The exact retrogressive failure solutions that will be constructed in $\S 5$ have a thickness profile that is almost identical to the dataset shown in figure $5(b)$.

\section{Governing equations}

\subsection{Depth-averaged avalanche model}

The retrogressive wave has a shallow aspect ratio (figure 5b) and is nearly uniform in the cross-slope direction (figure 3), which motivates the use of a one-dimensional depth-integrated avalanche model to describe the flow. The flow thickness $h(x, t)$ and depth-averaged downslope velocity $\bar{u}(x, t)$ therefore satisfy the depth-integrated mass and momentum equations

$$
\begin{gathered}
\frac{\partial h}{\partial t}+\frac{\partial}{\partial x}(h \bar{u})=0 \\
\frac{\partial}{\partial t}(h \bar{u})+\frac{\partial}{\partial x}\left(\chi h \bar{u}^{2}\right)+\frac{\partial}{\partial x}\left(\frac{1}{2} g h^{2} \cos \zeta\right)=g h S \cos \zeta
\end{gathered}
$$

where $x$ is the downslope coordinate, $t$ is time, $\chi=\overline{u^{2}} / \bar{u}^{2}$ is the shape factor, $\zeta$ is the chute inclination angle, $S$ is the non-dimensional net acceleration and $g$ is the constant 

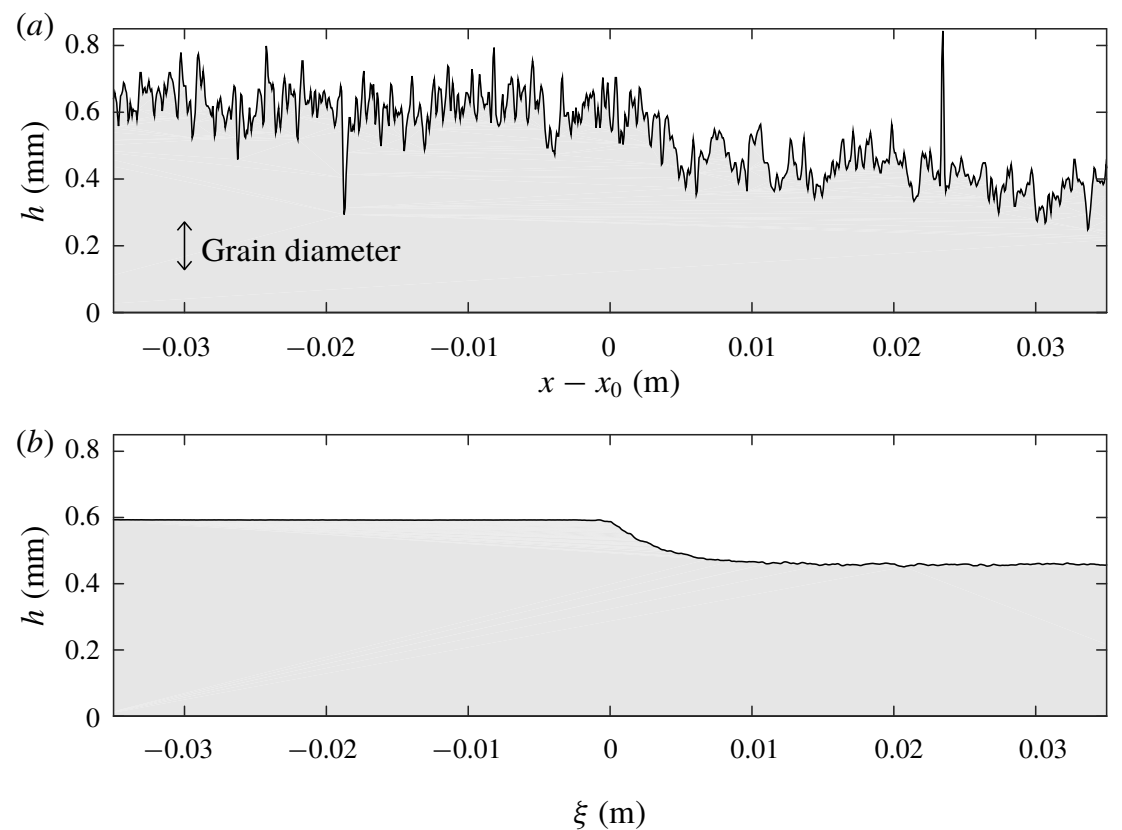

FIGURE 5. Measurements of the flow thickness $h$ during the retrogressive failure with an initial layer thickness $h_{0}=h_{\text {stop }}\left(25^{\circ}\right)=0.59 \mathrm{~mm}$ and current inclination angle $\zeta=27.5^{\circ}$. (a) An instantaneous snapshot of the thickness profile as a function of downslope distance $x-x_{0}$, where the constant $x_{0}$ is the position of the failure. The measured noise is of the order of one grain diameter. (b) Average of 1160 such profiles acquired at $1 \mathrm{~ms}$ intervals and plotted with respect to a coordinate $\xi=x-u_{w} t$, which moves upstream at constant speed $\left|u_{w}\right|=6.9 \mathrm{~cm} \mathrm{~s}^{-1}$.

of gravitational acceleration. For deep flows on rough beds, a steady uniform flow will develop into a Bagnold velocity profile (GDR-MiDi 2004; Gray \& Edwards 2014) and the shape factor $\chi=5 / 4$, while for thinner flows close to $h_{\text {stop }}$ weakly exponential profiles develop (Kamrin \& Henann 2015), which have a shape factor of approximately 1.53 for the typical profiles observed in our experiments. Solutions to this system are, however, quite insensitive to the value of the shape factor due to the low value of Froude number (Saingier, Deboeuf \& Lagrée 2016; Viroulet et al. 2017). Therefore it is assumed here that $\chi=1$, because it significantly simplifies the characteristic structure of the equations (see e.g. Savage \& Hutter 1989; Gray et al. 1999; Pouliquen 1999b; Gray et al. 2003; Pouliquen \& Forterre 2002; Gray \& Edwards 2014). The characteristic speeds of the hyperbolic system (3.1)-(3.2) are then

$$
c_{ \pm}=\bar{u} \pm \sqrt{g h \cos \zeta}
$$

and the ratio of flow speed to the gravity wave speed defines the Froude number

$$
F r=\frac{|\bar{u}|}{\sqrt{g h \cos \zeta}} .
$$

The non-dimensional net acceleration $S$ in the source term on the right-hand side of (3.2) is defined as the difference between the downslope component of gravity and 
the effective basal friction $\mu(h, F r)$

$$
S=\tan \zeta-\mu(h, F r)(\bar{u} /|\bar{u}|)
$$

where the factor $\bar{u} /|\bar{u}|$ ensures that the basal friction always opposes the direction of motion. In this paper the friction always acts upslope irrespective of whether the material is moving or static and hence $\bar{u} /|\bar{u}|=1$. The non-dimensional net acceleration $S$ determines whether a constant thickness layer will accelerate $(S>0)$, decelerate $(S<0)$ or move with constant speed $(S=0)$.

It is important to note that the depth-averaged viscous terms (Gray \& Edwards 2014; Baker, Barker \& Gray 2016a), which are crucial for the formation of leveed channels on non-erodible slopes (Rocha, Johnson \& Gray 2019), can be neglected here because the retrogressive failures are planar. It is, however, anticipated that viscous terms could well be important for non-planar retrogressive waves, where cross-slope gradients in the velocity will develop naturally, or, for the correct cutoff frequency and coarsening dynamics of roll waves and erosion-deposition waves that may form downstream of the failure front (Gray \& Edwards 2014; Edwards \& Gray 2015; Viroulet et al. 2018).

\subsection{The non-monotonic effective basal friction law}

Gray \& Edwards (2014) showed that, to leading order in the aspect ratio, both the inviscid avalanche equations (3.1)-(3.2) and the dynamic friction law of Pouliquen \& Forterre (2002) emerge naturally from depth averaging the $\mu(I)$-rheology for granular flows (GDR-MiDi 2004; Jop et al. 2006). In order to model coexisting regions of static and flowing material it is necessary to augment the dynamic regime $\left(F r \geqslant \beta_{*}\right)$ with the multi-valued static $(F r=0)$ and intermediate $\left(0<F r<\beta_{*}\right)$ regimes (Pouliquen \& Forterre 2002; Edwards et al. 2017, 2019). For glass ballotini (which does not have an offset $\Gamma$ in the empirical flow rule) the non-monotonic friction law of Edwards et al. (2019) reduces to

$$
\mu(h, F r)= \begin{cases}\mu_{1}+\frac{\mu_{2}-\mu_{1}}{1+h \beta /(\mathscr{L} F r)}, & F r \geqslant \beta_{*}, \\ \left(\frac{F r}{\beta_{*}}\right)^{\kappa}\left(\mu_{1}+\frac{\mu_{2}-\mu_{1}}{1+h \beta /\left(\mathscr{L} \beta_{*}\right)}-\mu_{3}-\frac{\mu_{2}-\mu_{1}}{1+h / \mathscr{L}}\right) & \\ +\mu_{3}+\frac{\mu_{2}-\mu_{1}}{1+h / \mathscr{L}}, & 0<F r<\beta_{*}, \\ \min \left(\mu_{3}+\frac{\mu_{2}-\mu_{1}}{1+h / \mathscr{L}},\left|\tan \zeta-\frac{\partial h}{\partial x}\right|\right), & F r=0,\end{cases}
$$

where $\mu_{i}=\tan \zeta_{i}$ for $i=1,2,3$ and the parameters are given in table 1. Importantly, this friction law is identical to that of Pouliquen \& Forterre (2002) when $F r>\beta_{*}$, and so is consistent with previous experimental measurements of steady uniform flows (Pouliquen 1999a; Pouliquen \& Forterre 2002).

The friction law (3.6)-(3.8) encodes information about the empirical flow rule (Pouliquen 1999a; Pouliquen \& Forterre 2002)

$$
F r=\beta \frac{h}{h_{\text {stop }}(\zeta)},
$$


$\zeta_{1}=21.27^{\circ}, \quad \zeta_{2}=33.89^{\circ}, \quad \zeta_{3}=25.3^{\circ}, \quad \beta=0.143, \quad \beta_{*}=0.19, \quad \mathscr{L}=0.2351 \mathrm{~mm}, \quad \kappa=1$.

TABLE 1. Parameters for the effective basal friction law (3.6)-(3.8) for $125-160 \mu \mathrm{m}$ diameter glass ballotini on a rough bed made of a monolayer of 750-1000 $\mu \mathrm{m}$ diameter glass ballotini. The angles $\zeta_{1-3}$ and $\mathscr{L}$ are fitted from the experimentally measured $h_{\text {stop }}(\zeta)$ and $h_{\text {start }}(\zeta)$ curves in figure $6, \beta$ is taken from Pouliquen $(1999 a)$ with a $1 / \sqrt{\cos \zeta}$ correction, while $\beta_{*}$ and $\kappa$ are measured using the retrogressive failure experiments in this paper.

and the $h_{\text {stop }}$ and $h_{\text {start }}$ curves

$$
\begin{array}{ll}
h_{\text {stop }}(\zeta)=\mathscr{L}\left(\frac{\tan \zeta_{2}-\tan \zeta_{1}}{\tan \zeta-\tan \zeta_{1}}-1\right), \quad \zeta \in\left[\zeta_{1}, \zeta_{2}\right], \\
h_{\text {start }}(\zeta)=\mathscr{L}\left(\frac{\tan \zeta_{2}-\tan \zeta_{1}}{\tan \zeta-\tan \zeta_{3}}-1\right), \quad \zeta \in\left[\zeta_{3}, \zeta_{4}\right],
\end{array}
$$

where $\zeta_{4}=\tan ^{-1}\left(\mu_{2}-\mu_{1}+\mu_{3}\right)$. Edwards et al. (2019) also introduced the minimum observable steady uniform flow thickness $h_{*}$, which occurs at the transition between the intermediate and dynamic regimes at $F r=\beta_{*}$. In particular, if $\beta_{*}$ is constant then the empirical flow rule (3.9) implies that $h_{*}$ is proportional to $h_{\text {stop }}$ (Edwards et al. 2019)

$$
h_{*}(\zeta)=\left(\frac{\beta_{*}}{\beta}\right) h_{\text {stop }}(\zeta) .
$$

In order to experimentally fit the $h_{\text {stop }}(\zeta)$ and $h_{\text {start }}(\zeta)$ functions (3.10) and (3.11) a steady uniform flow at an angle $\zeta_{0}$ is created, which leaves behind a deposit of $h_{\text {stop }}\left(\zeta_{0}\right)$. This static layer is then inclined gently to an angle $\zeta_{\text {start }}=h_{\text {start }}^{-1}\left(h_{\text {stop }}\left(\zeta_{0}\right)\right)>\zeta_{0}$, where it spontaneously starts flowing. There is a large spread in the data for $\zeta_{\text {start }}$ due to the layer of grains becoming increasingly sensitive to infinitesimal perturbations as it is inclined towards the angle at which it will spontaneously fail. The $\zeta_{\text {start }}$ curve is also sensitive to the packing of the grains, which induces some intrinsic variation in the data (Balmforth \& McElwaine 2018). This process is repeated for a range of angles $\zeta_{0}$ to generate the data in figure 6 and hence determine the parameters $\zeta_{1}, \zeta_{2}, \zeta_{3}$ and $\mathscr{L}$ in table 1 . In this paper, the value of $\beta$ is essentially taken to be the same as the value of 0.136 measured in experiments of Pouliquen (1999a) for glass ballotini. However, due to Pouliquen's (1999a) alternative definition of the Froude number $F r=$ $|\bar{u}| / \sqrt{g h}$, compared to (3.4), a correction of $1 / \sqrt{\cos \zeta}$ is made, with a typical value of $\zeta$ in the range $\left[22^{\circ}, 28^{\circ}\right]$ studied by Pouliquen $(1999 a)$, to give $\beta=0.143$.

The formula for the intermediate regime (3.7) is a monotonically decreasing function of the Froude number, which interpolates between the maximum static friction $\mu_{\text {start }}$ and the minimum dynamic friction at $F r=\beta_{*}$ (Pouliquen \& Forterre 2002; Edwards et al. 2017, 2019). Edwards et al. (2017, 2019) suggested an interpolation power $\kappa=1$ in (3.7) to give static material in the metastable range of thicknesses greater stability to small perturbations than in Pouliquen \& Forterre's (2002) original formulation. Pouliquen \& Forterre (2002) used a value of $\kappa=10^{-3}$ instead, which implies that the hysteretic friction is only partially represented in typical machine precision calculations (Edwards et al. 2019) and as a result, the metastable range 


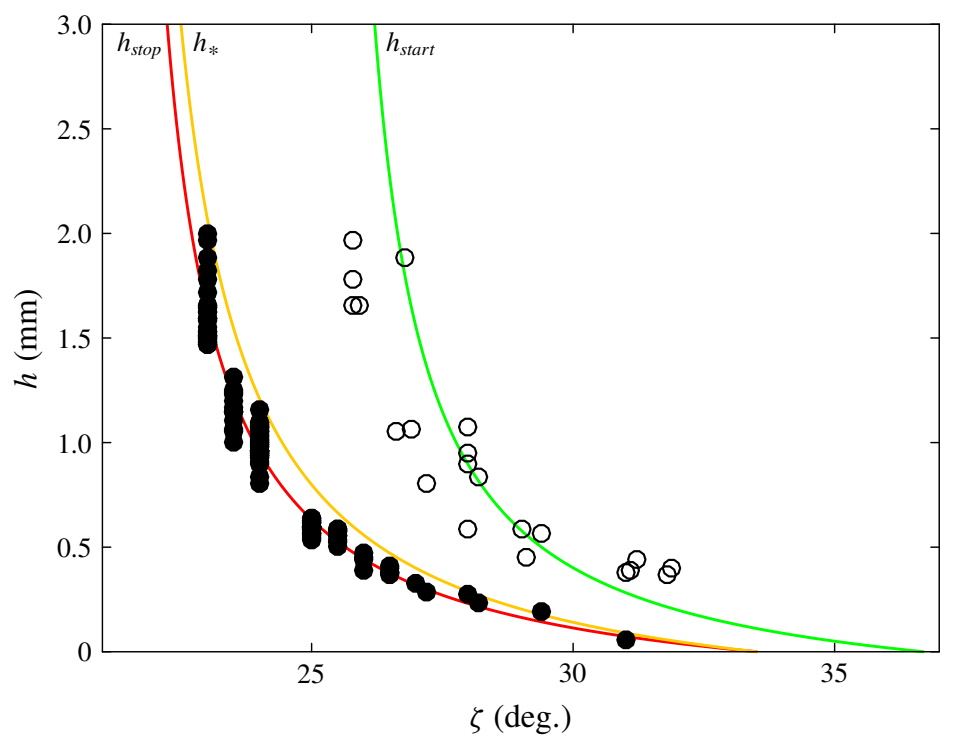

FIGURE 6. Experimental data for the $h_{\text {stop }}(\zeta)$ and $h_{\text {start }}(\zeta)$ curves (black and white circles, respectively) and empirical fits (red and green lines, respectively) using equations (3.10) and (3.11) and the parameters in table 1. The experiments are performed with $125-160 \mu \mathrm{m}$ spherical glass ballotini on a monolayer of $750-1000 \mu \mathrm{m}$ spherical glass beads stuck to a wooden surface. The orange line shows the functional form of $h_{*}(\zeta)$ given by (3.12).

of thicknesses $\left[h_{*}, h_{\text {start }}\right]$ is much more sensitive to disturbances than is physically realistic.

For the glass beads in our experiments, the slowest steady uniform flows attainable are at $h_{*} / h_{\text {stop }}=1.33$, which by (3.12) implies $\beta_{*}=0.19$ and hence that $h_{*} \in\left(h_{\text {stop }}, h_{\text {start }}\right)$ for all angles in $\left[\zeta_{1}, \zeta_{2}\right]$. The friction transition at $\mathrm{Fr}=\beta_{*}$ implies the minimum steady uniform flow thickness $h_{*}>h_{\text {stop }}$, contrary to Pouliquen \& Forterre's (2002) original hypothesis that the minimum steady uniform flow thickness $h_{\text {stop }}$ occurred at $F r=\beta$. Edwards et al. (2017, 2019) showed, however, that modifying the transition in this way implied that a steady uniform flow close to $h_{*}$ produced a deposit depth close to $h_{\text {stop }}$ as observed in experiment, whilst Pouliquen \& Forterre's (2002) transition produced deposits that were thinner than $h_{\text {stop }}$ (see e.g. Edwards \& Gray 2015).

\section{Numerical simulations of retrogressive failure}

Retrogressive failure fronts are simulated by solving the conservation laws (3.1)(3.2) together with the friction law (3.6)-(3.8) numerically. The central scheme of Kurganov \& Tadmor (2000) and a second-order Runge-Kutta method are used to discretise the equations, with the time step determined by a Courant-Friedrichs-Lewy (CFL) number of $1 / 4$. In static regions, the equilibrium force balance is assessed prior to each time step and the friction coefficient set appropriately using (3.8), resulting in a well-balanced discretisation of the source terms that preserves these static states exactly. The material parameter values used in these numerical simulations are given in table 1. 

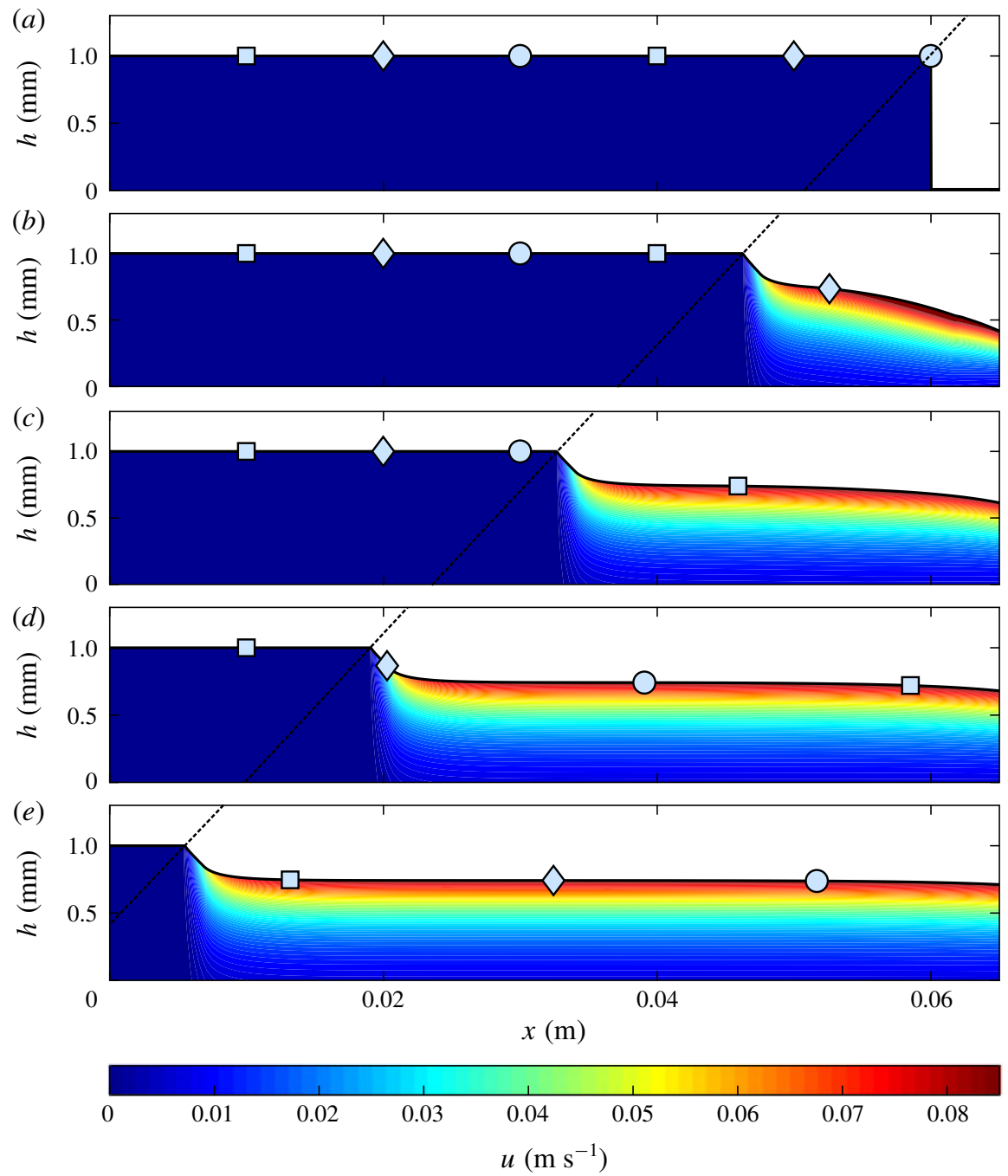

FIGURE 7. The retrogressive wave thickness $h(x, t)$ and downslope velocity $u(x, z, t)$ at a sequence of time steps $(a) 0 \mathrm{~s}(b) 0.15 \mathrm{~s}(c) 0.3 \mathrm{~s}(d) 0.45 \mathrm{~s}$ and $(e) 0.6 \mathrm{~s}$ for a slope inclined at $\zeta=27.5^{\circ}$. The initial stationary layer is of thickness $h(x, 0)=1.0 \mathrm{~mm}$. The filled region shows the thickness and the contour scale within it denotes the velocity, which is reconstructed from the depth-averaged downslope velocity $\bar{u}(x, t)$ assuming an exponential profile (4.1) with $\lambda=2.45$. There is no inflow at $x=0$ and there is free outflow at the downstream boundary. The diagonal dashed line shows that the retrogressive failure front rapidly develops and travels upslope at a constant speed. The wave erodes the static surface particles, which are shown with light blue markers and they travel downslope on the surface of the steady uniform flow. The material properties are given in table 1 and an animation is shown in movie 5 of the online supplementary material.

A solution exhibiting retrogressive failure on a slope inclined at $\zeta=27.5^{\circ}$ is shown in figure 7 and supplementary movie 5. Initially for $x<0.06 \mathrm{~m}$ there is a stationary layer of thickness $h(x, 0)=1.0 \mathrm{~mm}$ and for $x>0.06 \mathrm{~m}$ the chute is empty. There is no inflow at $x=0$ and free outflow at the downstream boundary. A retrogressive failure 
wave rapidly develops (figure $7 b$ ) and travels upslope at a constant speed (figure $7 b-e$, indicated by the dashed line). This wave connects a thicker static deposit upslope and thinner flowing region downslope.

The two-dimensional downslope velocity field $u(x, z, t)$ is reconstructed from the depth-averaged downslope velocity $\bar{u}(x, t)$ assuming an exponential velocity profile through the depth of the avalanche as in Wiederseiner et al. (2011),

$$
u(x, z, t)=\frac{\lambda \bar{u}(x, t)}{\exp (\lambda)-1} \exp \left(\frac{\lambda z}{h}\right)
$$

where the non-dimensional parameter $\lambda$ determines the ratio of surface to depthaveraged velocity,

$$
\frac{u_{s}}{\bar{u}}=\frac{\lambda \exp (\lambda)}{\exp (\lambda)-1}
$$

Concave velocity profiles of this sort are predicted for shallow flows by the nonlocal rheology of Kamrin \& Henann (2015) and by discrete element simulations (their figure 2).

The parameter $\lambda$ is calibrated experimentally by creating a static layer at an angle $\zeta_{0}$, placing a ruler across the deposit and sweeping the grains off the chute downslope of the ruler. The chute was then inclined to an angle $\zeta>\zeta_{0}$ and the ruler was then removed, allowing a retrogressive failure to propagate upslope through the static layer of grains. Downstream of the release a steady uniform flow rapidly developed with a granular front (Pouliquen 1999b) that propagated downslope with the depth-averaged speed of the flow. Both the surface velocity $u_{s}$ of the uniform flow and the speed of the granular front $\bar{u}$ were measured, and (4.2) was used to determine $\lambda$. For experimental conditions close to those in figure 4 the surface velocity $u_{s}=8.5 \mathrm{~cm} \mathrm{~s}^{-1}$ and $\bar{u}=3.2 \mathrm{~cm} \mathrm{~s}^{-1}$, giving $u_{s} / \bar{u}=2.7$ and hence $\lambda=2.45$. This reconstruction allows integration of surface particle trajectories (light blue markers in figure 7). As the particles accelerate and the flow thins, the spacing between the particles increases. An animation showing how the particles move at different depths in the flow (movie 6) is available in the online supplementary material.

The downslope surface velocity can also be visualised in a space-time plot (figure 8). The contours of $u_{s}$ form parallel diagonal lines, indicating the rapid development of a travelling retrogressive failure that moves upslope at a constant speed. A series of particle trajectories are plotted on top of the contours (light blue lines), with stationary particles upslope of the failure (horizontal lines) and moving particles downslope (diagonal lines). This is the same behaviour shown in the experimental space-time plot in figure 4. The simulated retrogressive wave speed of $9.1 \mathrm{~cm} \mathrm{~s}^{-1}$ is similar to the experimentally measured wave speed $\left|u_{w}\right|=8.5 \mathrm{~cm} \mathrm{~s}^{-1}$, while the simulated surface velocity of the steady uniform flow downstream of the failure $u_{s}=8.1 \mathrm{~cm} \mathrm{~s}^{-1}$ is within $0.1 \mathrm{~cm} \mathrm{~s}^{-1}$ of that measured in the experiment.

\section{Travelling wave solutions for retrogressive failure}

The experiments in $\S 2$ and the numerical simulations in $\S 4$ suggest that retrogressive failures rapidly develop into travelling waves. This is now investigated, within the framework of the avalanche model described in $\S 3$, by using a travelling wave ansatz. 


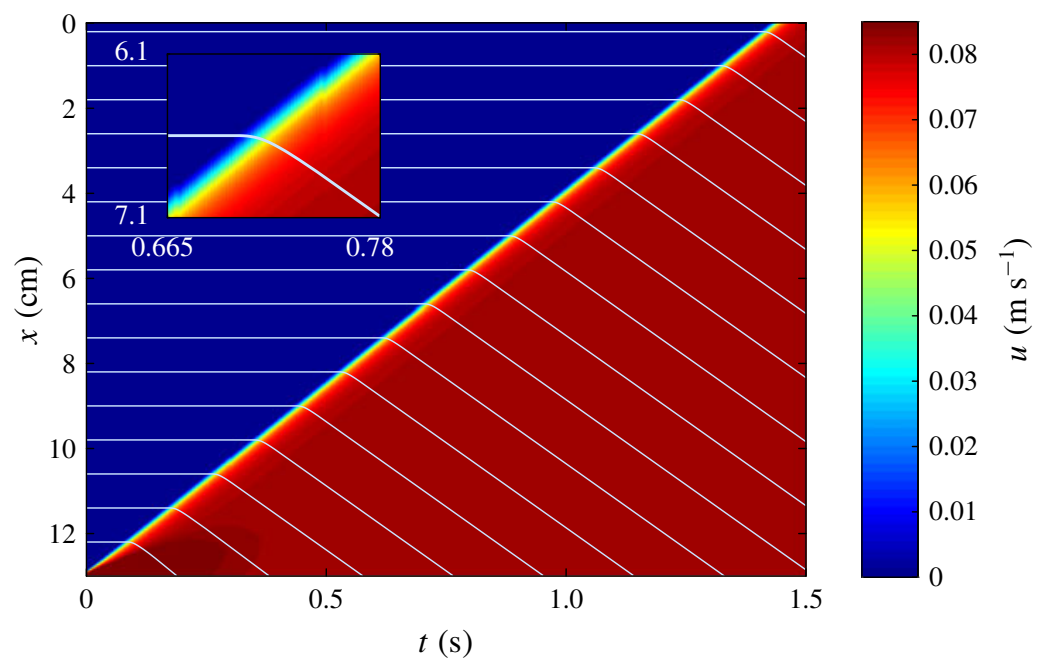

FIgURE 8 . Space-time $(t, x)$ plot showing the surface velocity $u(x, h, t)$ of a retrogressive failure front on a slope inclined at $\zeta=27.5^{\circ}$ with an initial stationary layer of thickness $h(x, 0)=1.0 \mathrm{~mm}$. Individual surface particles are tracked (light blue lines) to visualise the particle trajectories through the retrogressive failure front. The horizontal lines indicate stationary particles and the diagonal lines represent moving surface particles whose velocity is reconstructed assuming an exponential profile (4.1) with $\lambda=2.45$. The inset shows a close up of one of the particle paths as it goes through the retrogressive failure front. The material properties are given in table 1 .

\subsection{Equations in a steadily moving frame}

A schematic diagram of the anticipated solution structure is shown in figure 1 . It consists of a static region of constant thickness $h_{0}$, that lies upstream of a flowing region, which decreases in thickness as it accelerates towards a steady uniform flow of thickness $h_{\infty}<h_{0}$ and depth-averaged velocity $\bar{u}_{\infty}$. The retrogressive failure is assumed to travel upslope with velocity $u_{w}<0$. Looking for steady solutions in the frame of the retrogressive wave $\xi=x-u_{w} t$, the governing equations (3.1)-(3.2) reduce to

$$
\begin{gathered}
\frac{\mathrm{d}}{\mathrm{d} \xi}\left(h\left(\bar{u}-u_{w}\right)\right)=0, \\
\left(\bar{u}-u_{w}\right) \frac{\mathrm{d} \bar{u}}{\mathrm{~d} \xi}+g \cos \zeta \frac{\mathrm{d} h}{\mathrm{~d} \xi}=g S \cos \zeta,
\end{gathered}
$$

where the acceleration terms in (5.2) have been simplified using (5.1). Integrating the mass balance (5.1) with respect to $\xi$, subject to the condition that the thickness is constant $h=h_{0}$ and $\bar{u}=0$ in the static region, implies that the flux in the moving frame is constant

$$
h\left(\bar{u}-u_{w}\right)=-u_{w} h_{0} .
$$

This can be rearranged to show that the depth-averaged velocity, anywhere in the flow, is a function of the wave velocity and thickness

$$
\bar{u}=u_{w}\left(1-\frac{h_{0}}{h}\right) \text {. }
$$


Substituting for the depth-averaged velocity in (5.2) and using (5.3) to simplify the result, implies that the thickness profile satisfies the autonomous ordinary differential equation (ODE)

$$
\frac{\mathrm{d} h}{\mathrm{~d} \xi}=\frac{S(h)}{1-\frac{u_{w}^{2} h_{0}^{2}}{g h^{3} \cos \zeta}},
$$

where $S(h)=\tan \zeta-\mu(h, \operatorname{Fr}(h))$ is defined in (3.5) and the Froude number can be written using (3.4) and (5.4) in terms of the thickness, initial thickness and wave speed, as

$$
F r=\frac{-u_{w}}{\sqrt{g h \cos \zeta}}\left(\frac{h_{0}}{h}-1\right) .
$$

The initial layer depth $h_{0}$ and the inclination $\zeta$ are prescribed in experiments, so equation (5.5) is a first-order ODE for $h$ with the wave velocity $u_{w}$ a free parameter. As the flow thins from the initial thickness $h_{0}$ to the steady uniform thickness $h_{\infty}$ the Froude number increases from zero to the steady uniform flow Froude number $F r_{\infty}=\bar{u}_{\infty} / \sqrt{g h_{\infty} \cos \zeta}$. This must lie in the dynamic frictional regime, since steady uniform flows in the intermediate regime are unstable. However, in order to connect the static and dynamic equilibria the solution passes through a point where $S=0$ in the intermediate regime (illustrated in figure 9); this must necessarily occur since the Froude number (5.6) is a strictly decreasing function of $h$. If the denominator on the right-hand side of (5.5) is non-zero, solutions to the ODE (5.5) have zero gradient where $S=0$, preventing a solution that connects the static and dynamic equilibrium solutions. Consequently, the denominator must be zero when $S=0$ in the intermediate regime, forming a critical point of the ODE at

$$
h=h_{c}=\left(\frac{u_{w}^{2} h_{0}^{2}}{g \cos \zeta}\right)^{1 / 3} .
$$

Writing the ODE (5.5) as

$$
\frac{\mathrm{d} h}{\mathrm{~d} \xi}=\frac{S(h)}{1-\left(h_{c} / h\right)^{3}},
$$

and using L'Hôpital's rule gives a finite value for the gradient at the critical point,

$$
\left.\frac{\mathrm{d} h}{\mathrm{~d} \xi}\right|_{h=h_{c}}=\left.\frac{h_{c}}{3} \frac{\mathrm{d} S}{\mathrm{~d} h}\right|_{h=h_{c}} .
$$

Although $h_{0}$ is given, it is easiest to solve the problem by assuming a value of the critical thickness $h_{c}$ and then solving for $h_{c}=h_{c}\left(h_{0}\right)$ later. Assuming that $h_{c}$ is given, the critical Froude number at $h=h_{c}$, can be found by solving $S=0$ using (3.5) and the intermediate friction law (3.7) to give

$$
F r_{c}=\beta_{*}\left[\frac{\tan \zeta-\mu_{3}-\frac{\mu_{2}-\mu_{1}}{1+h_{c} / \mathscr{L}}}{\mu_{1}+\frac{\mu_{2}-\mu_{1}}{1+h_{c} \beta / \mathscr{L} \beta_{*}}-\mu_{3}-\frac{\mu_{2}-\mu_{1}}{1+h_{c} / \mathscr{L}}}\right]^{1 / \kappa} .
$$




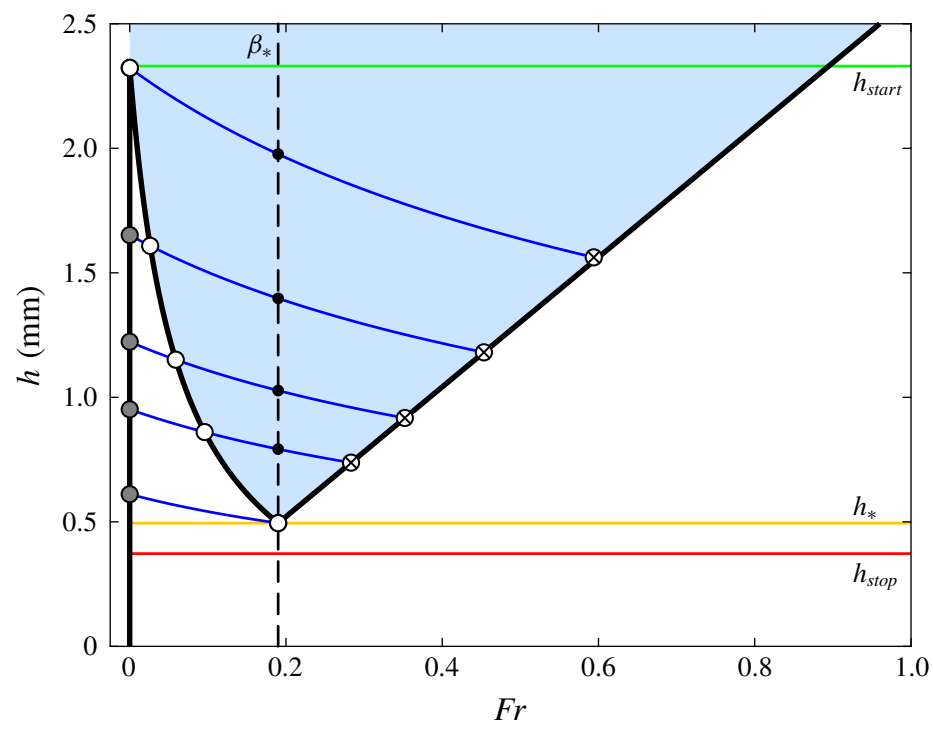

FIgURE 9. Contour $S=0$ (thick black line) as a function of the Froude number $\mathrm{Fr}$ and the thickness $h$ for a slope angle $\zeta=26.5^{\circ}$. In the blue shaded region a constant depth flow is accelerative $(S>0)$, in the white region it is decelerative $(S<0)$ and on the zero contour $(S=0)$ it moves at constant speed. The green line is $h_{\text {start }}$, the orange line is $h_{*}$ and the red line is $h_{\text {stop }}$. The vertical dashed line lies at $F r=\beta_{*}$, which is where the friction switches from the intermediate to the dynamic regime. The blue lines show solutions to the ODE (5.5) for different initial layer thicknesses $h_{0}$ and which are parameterised by the curve (5.6) with different values of $u_{w}$. The white circles are the critical points, the grey circles are the transitions to static friction, the black circles are the transitions to dynamic friction and the white circles containing the crosses are the steady uniform flow velocities downstream $\left(h_{\infty}, \bar{u}_{\infty}\right)$.

From the definition of the Froude number (3.4) it follows that the critical velocity

$$
\bar{u}_{c}=F r_{c} \sqrt{g h_{c} \cos \zeta} .
$$

Substituting $h_{c}$ and $\bar{u}_{c}$ into the integrated mass balance (5.3) implies that

$$
h_{0} u_{w}=h_{c}\left(u_{w}-\bar{u}_{c}\right) .
$$

Solving (5.7) gives another expression for $h_{0} u_{w}$,

$$
h_{0} u_{w}=-h_{c} \sqrt{g h_{c} \cos \zeta},
$$

where the negative root in the quadratic is assumed, so that $u_{w}$ is negative. Equating (5.12) and (5.13) then determines the velocity of the retrogressive wave

$$
u_{w}=\bar{u}_{c}-\sqrt{g h_{c} \cos \zeta},
$$

which is equal to the upward characteristic velocity (3.3) evaluated at the critical point. Substituting the wave velocity (5.14) back into (5.13) and dividing by $\sqrt{g h_{c} \cos \zeta}$ then determines the corresponding initial deposit thickness

$$
h_{0}=\frac{h_{c}}{1-F r_{c}} .
$$



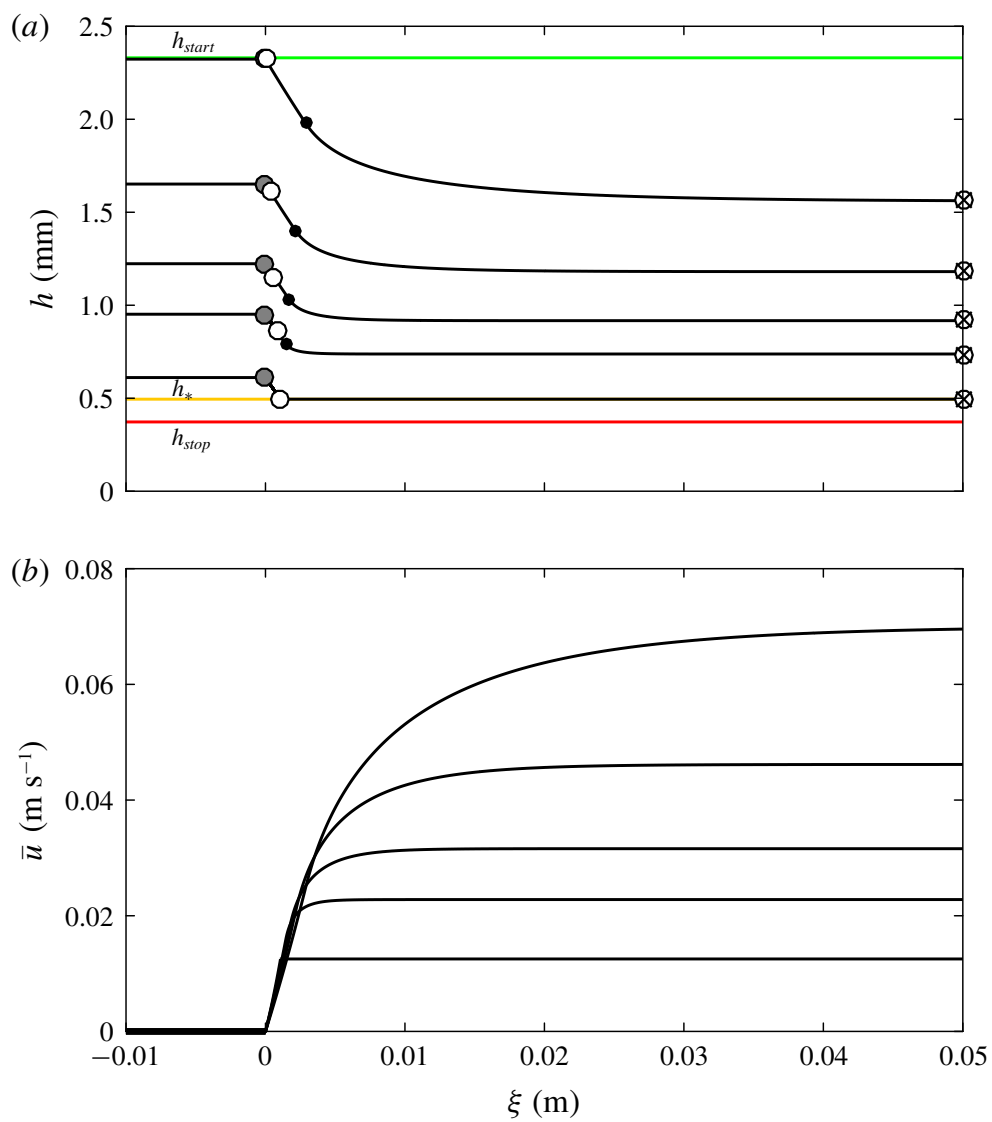

FIGURE 10. A series of retrogressive travelling wave solutions for $(a)$ the thickness $h$ and $(b)$ the depth-averaged downslope velocity $\bar{u}$ as a function of the travelling wave coordinate $\xi$ and for an inclination $\zeta=26.5^{\circ}$. The solutions correspond to an initial layer thicknesses $h_{0}=h_{\text {stop }}\left(22.55^{\circ}\right), h_{\text {stop }}\left(23^{\circ}\right), h_{\text {stop }}\left(23.5^{\circ}\right), h_{\text {stop }}\left(24^{\circ}\right), h_{\text {stop }}\left(25.068^{\circ}\right)$, with the thicker layers corresponding to $h_{\text {stop }}$ at lower angles. The point at which static material is mobilised by the wave at $\xi=0$ is indicated by a grey filled circle, the critical point $h=h_{c}$ by a white filled circle, the transition to dynamic friction at $F r=\beta_{*}$ by a black circle and the steady uniform flow downstream $\left(h=h_{\infty}, \bar{u}=\bar{u}_{\infty}\right)$ by a crossed circle. The deeper steady uniform flows in $(a)$ correspond to faster depth-averaged velocities in $(b)$ and there is no motion for $\xi<0$.

If, as in the experiment, the initial deposit thickness $h_{0}$ is known but $h_{c}$ is not, $h_{c}$ can be found by inverting (5.15) numerically with $F r_{c}\left(h_{c}\right)$ evaluated using (5.10). For a given value of the thickness $h_{c}$, it follows that the Froude number $F r_{c}$, the velocity at the critical point $\bar{u}_{c}$, the wave velocity $u_{w}$ and the initial deposit thickness $h_{0}$ are determined by (5.10), (5.11), (5.14) and (5.15), respectively. The thickness profile $h(\xi)$ can then be calculated by numerically integrating (5.8) upslope from $h_{c}$ to $h_{0}$ and downslope from $h_{c}$ to $h_{\infty}$, using L'Hôpital's rule (5.9) to start the integration.

A series of thickness profiles for different static deposit thicknesses $h_{0}$ and for $\zeta=26.5^{\circ}$ are shown in figure $10(a)$, aligned as in figure $5(b)$ so that the point of failure lies at $\xi=0$. Upslope of the failure point, the material is stationary and of constant thickness $h_{0}$, whilst downslope of it the thickness decreases and tends 
towards the steady uniform flow thickness $h_{\infty}$ as $\xi \rightarrow \infty$. At $\xi=0$ there is a discontinuity in $\mathrm{d} h / \mathrm{d} \xi$, which is consistent with the experimental observations in figure $5(b)$. This is due to the discontinuity in friction between the static friction $\mu=\tan \zeta$ (3.8) and intermediate friction (3.7) at the point of failure. In the flowing region, the depth-averaged velocity is given by (5.4) and rises smoothly from zero at $\xi=0$ towards the steady uniform flow velocity $\bar{u}_{\infty}$ as $\xi \rightarrow \infty$ (figure $10 b$ ). For deeper initial layers the steady uniform flow thickness $h_{\infty}$ and the velocity $\bar{u}_{\infty}$ are greater than for thinner layers. The length scale for the transition from the static to the flowing state is an increasing function of both the initial layer thickness $h_{0}$ and inclination angle $\zeta$.

The flow far downstream can be found without numerical integration of the governing ODE. The dynamic friction law (3.6) implies that the Froude number far downstream is

$$
F r_{\infty}=\left(\frac{\tan \zeta-\mu_{1}}{\mu_{2}-\tan \zeta}\right) \frac{\beta h_{\infty}}{\mathscr{L}},
$$

which, by using its definition (3.4), allows the velocity $\bar{u}_{\infty}$ to be calculated as a function of $h_{\infty}$. Integrating the transformed mass balance (5.1), but evaluating the constant of integration at the critical point rather than in the static layer, implies that

$$
h_{\infty}\left(\bar{u}_{\infty}-u_{w}\right)=h_{c}\left(\bar{u}_{c}-u_{w}\right) .
$$

Solving (5.16) and (5.17) determines the thickness far downstream for a given critical thickness $h_{c}$, since $u_{w}$ and $\bar{u}_{c}$ are known from (5.14) and (5.11), respectively.

The solutions can also be visualised in $(F r, h)$-space as shown in figure 9 . The solutions connect the static layer to the steady uniform flow solution far downslope with a trajectory that smoothly passes through the critical point $\left(F r_{c}, h_{c}\right)$ on the $S=0$ contour in the intermediate friction regime.

\subsection{Existence of retrogressive travelling wave solutions}

The retrogressive wave solutions outlined in $\$ 5.1$ exist over a range of slope angles $\zeta$ and initial layer thicknesses $h_{0}$. An upper bound for the initial layer thickness is provided by the requirement that the static layer does not fail spontaneously, which implies that

$$
h_{0}<h_{\text {start }}(\zeta)=h_{0}^{\max }(\zeta)
$$

The function $h_{\text {start }}(\zeta)$ takes a finite value when $\zeta>\zeta_{3}$ as in figure 9 , but is infinite for shallower inclinations $\zeta \leqslant \zeta_{3}$, in which case the initial static layer may be arbitrarily thick. A lower bound for the initial thickness $h_{0}$ stems from the requirement that a steady uniform flow exists far downstream, which implies that $h_{\infty} \geqslant h_{*}$ as shown in figures 9 and 10. It follows that the minimum initial thickness $h_{0}$ for which a solution exists occurs when $h_{c}=h_{*}=h_{\infty}$ and $F r=\beta_{*}$, which, using (5.15) and (3.12), implies that

$$
h_{0}^{\text {min }}=\frac{h_{*}}{1-\beta_{*}}=\frac{\beta_{*}}{\left(1-\beta_{*}\right) \beta} h_{\text {stop }}(\zeta),
$$

which is a constant multiple of $h_{\text {stop }}(\zeta)$. The upper and lower bounds (5.18) and (5.19) define a region in phase space, shown in figure 11, where retrogressive waves 


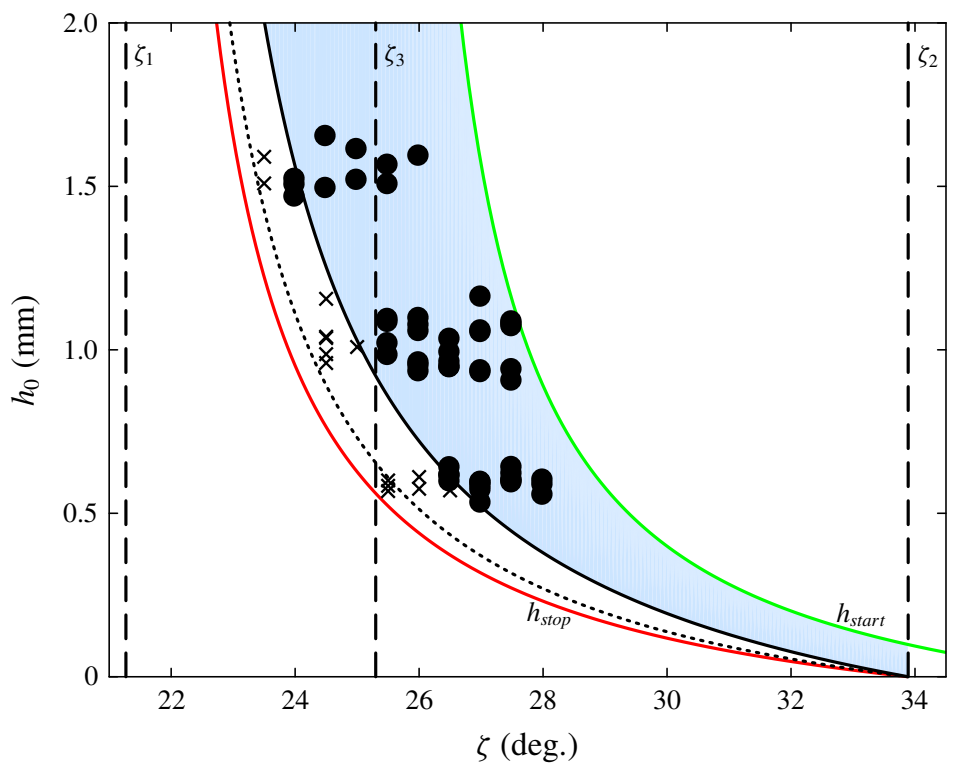

FIGURE 11. Experimental phase diagram showing the values of slope angle $\zeta$ and static layer thickness $h_{0}$ for which retrogressive failures are observed (black circles) and are not observed (crosses). This agrees well with the predicted domain of existence for $h_{0} \in\left[h_{0}^{\min }(\zeta), h_{\text {start }}(\zeta)\right)$, which is shaded blue. In particular, the lower boundary $h_{0}^{\min }(\zeta)$ (solid black curve) is in much better agreement than when $\beta_{*}=\beta$ (dotted curve), which corresponds to Pouliquen \& Forterre's (2002) original friction law. The red line represents $h_{\text {stop }}(\zeta)$, the green line to $h_{\text {start }}(\zeta)$ and the vertical dashed lines correspond to the angles $\zeta_{1}, \zeta_{2}$ and $\zeta_{3}$.

exist. Experiments recording the existence or not of a retrogressive wave over a range of $\zeta$ and $h_{0}$ are in good agreement with the theoretical predictions (figure 11). Below $h_{0}^{\min }(\zeta)$ retrogressive failures are not observed, but avalanches can propagate downslope and in some cases, for initial layer thicknesses slightly greater than $h_{0}^{\min }(\zeta)$, erosion-deposition waves (Edwards \& Gray 2015) can form downstream of the retrogressive failure. This experimental determination of $h_{0}^{\min }(\zeta)$ supports a constant value of $\beta_{*}$ for all inclinations (as suggested by Edwards et al. 2019) with $\beta_{*}=0.19 \approx 1.33 \beta$, corresponding to a minimum thickness of steady uniform flow $h_{*} \approx 1.33 h_{\text {stop }}$. The prediction for $h_{0}^{\min }$ with $\beta_{*}=\beta$ (dotted line in figure 11 , as suggested by Pouliquen \& Forterre 2002) is not in such good agreement with our experiments. The experimental phase boundary $h_{0}^{\min }(\zeta)$ therefore provides an important constraint on the theory that also helps to determine $\beta_{*}$.

Retrogressive waves are predicted for angles up to $\zeta_{4}=\tan ^{-1}\left(\mu_{3}+\mu_{2}-\mu_{1}\right)$, the angle at which $h_{\text {start }}$ reaches zero. For $\zeta>\zeta_{2}$, the method to determine the critical point and the retrogressive wave speed remains unchanged, but the flow accelerates and thins indefinitely downslope once it has failed. This regime is not observed in our experiments, because the predicted static layer depths are too small $(\sim 1$ grain diameter), but such solutions may be observable in grains with a larger static friction coefficient that can form thicker static layers at steep inclinations $\zeta>\zeta_{2}$. 


\subsection{Retrogressive wave speed}

The theoretical wave speed $\left|u_{w}\right|$ is compared with the experimentally measured values in figure 12 for a range of slope angles $\zeta$ and for initial layers of thickness (a) $h_{0}=h_{\text {stop }}\left(24^{\circ}\right)=0.95 \mathrm{~mm}$ and $\left(\right.$ b) $h_{0}=h_{\text {stop }}\left(25^{\circ}\right)=0.63 \mathrm{~mm}$. Since both $h_{\text {start }}(\zeta)$ and $h_{\text {stop }}(\zeta)$ are monotonically decreasing functions, the upper and lower bounds (5.18) and (5.19) can be rearranged to give the maximum and minimum slope angles at which an initial layer of thickness $h_{0}$ will form a retrogressive wave

$$
\begin{gathered}
\zeta^{\max }=\zeta_{\text {start }}\left(h_{0}\right), \\
\zeta^{\min }=\zeta_{\text {stop }}\left(\frac{\left(1-\beta_{*}\right) \beta}{\beta_{*}} h_{0}\right),
\end{gathered}
$$

where $\zeta_{\text {stop }}(h)$ and $\zeta_{\text {start }}(h)$ are the inverse functions of $h_{\text {stop }}(\zeta)$ and $h_{\text {start }}(\zeta)$, respectively. For angles $\zeta>\zeta^{\max }$ the initial layer is thicker than $h_{\text {start }}$ and therefore spontaneously flows off the inclined plane, while for $\zeta<\zeta^{\text {min }}$ a retrogressive wave does not propagate upslope, although an avalanche forms downstream of the perturbation. In both of these situations the retrogressive wave speed is defined to be zero.

For the two sets of experiments in figure 12 the range of angles over which retrogressive failures are, and are not, observed agree well with the theoretically predicted limits $\zeta^{\min }$ and $\zeta^{\max }$. In the range of angles where retrogressive waves are observed both sets of data show that the wave speed increases with increasing inclination angle. This trend is well predicted by the theory, but the magnitude of the wave speed is slightly under-predicted. The theory does, however, predict the immediate jump from zero up to a finite non-zero wave speed at the onset of retrogressive failures at $\zeta=\zeta^{\min }$. The scatter in the experimental data points is primarily due to the difficulty of precisely controlling the initial static layer thickness $h_{0}$.

The theoretical wave velocity (5.14) is dependent on the thickness $h_{c}$ and the depthaveraged velocity $\bar{u}_{c}$ at the critical point. Substituting for $\bar{u}_{c}$ from (5.11) it follows that the wave speed $\left|u_{w}\right|$ can be written as

$$
\left|u_{w}\right|=\left(1-F r_{c}\right) \sqrt{g h_{c} \cos \zeta},
$$

where $F r_{c}\left(h_{c}\right)$ is defined in (5.10). Experimental measurements of the wave speed therefore provide indirect experimental evidence for the form of the friction law in the intermediate regime $0<F r<\beta_{*}$. This evidence is valuable because steady uniform flows, which are widely used to determine the flow rule for $\mathrm{Fr}>\beta_{*}$ (Pouliquen 1999a), are unstable in intermediate regime $0<F r<\beta_{*}$ and so cannot be observed experimentally. The friction in the intermediate regime (3.7) is a power-law interpolation between the maximum static friction at $F r=0$ and the minimum dynamic friction at $F r=\beta_{*}$, with exponent $\kappa$ that is taken to be unity (figure $13 a$, inset). This form of the friction coefficient results in the retrogressive wave speed being an increasing function of the slope angle for a given layer thickness $h_{0}$ (figure 13a), which is in agreement with experimental cases shown in figure $12(a, b)$.

In Pouliquen \& Forterre's (2002) original friction law the interpolation parameter was chosen to be very small (e.g. $\kappa=10^{-3}$ ), which corresponds to a rapid decrease from the maximum static friction at low Froude numbers, as shown in the inset in figure 13(b). This choice of interpolation leads to an extremely small critical Froude number $F r_{c}$, and consequently $h_{c} \approx h_{0}$. It follows from (5.22) that the wave speed

$$
\left|u_{w}\right| \approx \sqrt{g h_{0} \cos \zeta} .
$$



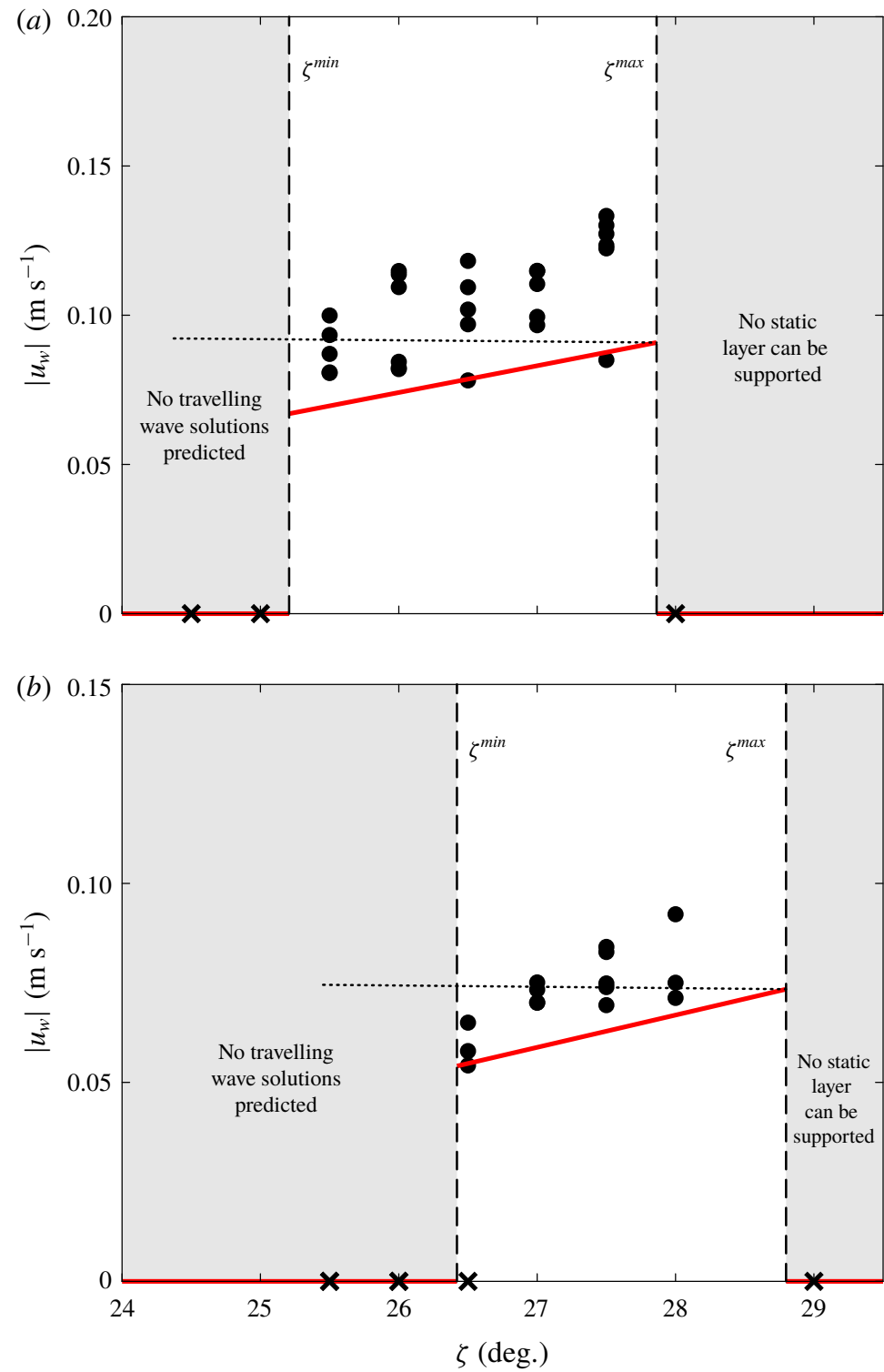

FIGURE 12. The retrogressive wave speed $\left|u_{w}\right|$ for $(a) h_{0}=h_{\text {stop }}\left(24^{\circ}\right)=0.95 \mathrm{~mm}$ and (b) $h_{0}=h_{\text {stop }}\left(25^{\circ}\right)=0.63 \mathrm{~mm}$ as a function of inclination angle $\zeta$. The circles represent experimental data for the retrogressive front and the crosses show the cases when either (i) there is no retrogressive failure, although there is an avalanche that propagates downslope, or (ii) a static layer of thickness $h_{0}$ can no longer be supported. The red line represents the theoretical prediction of the wave speed $\left|u_{w}\right|$. The black dotted line corresponds to the prediction from Pouliquen \& Forterre's (2002) original friction law (5.23). Note that since $\beta_{*}=\beta$ these lines extend considerably into the region where retrogressive failures are not observed.

This is a slightly decreasing function of the inclination angle $\zeta$ (see the dotted lines in figure 12 as well as figure $13 b$ ), which is the opposite trend to that observed in 

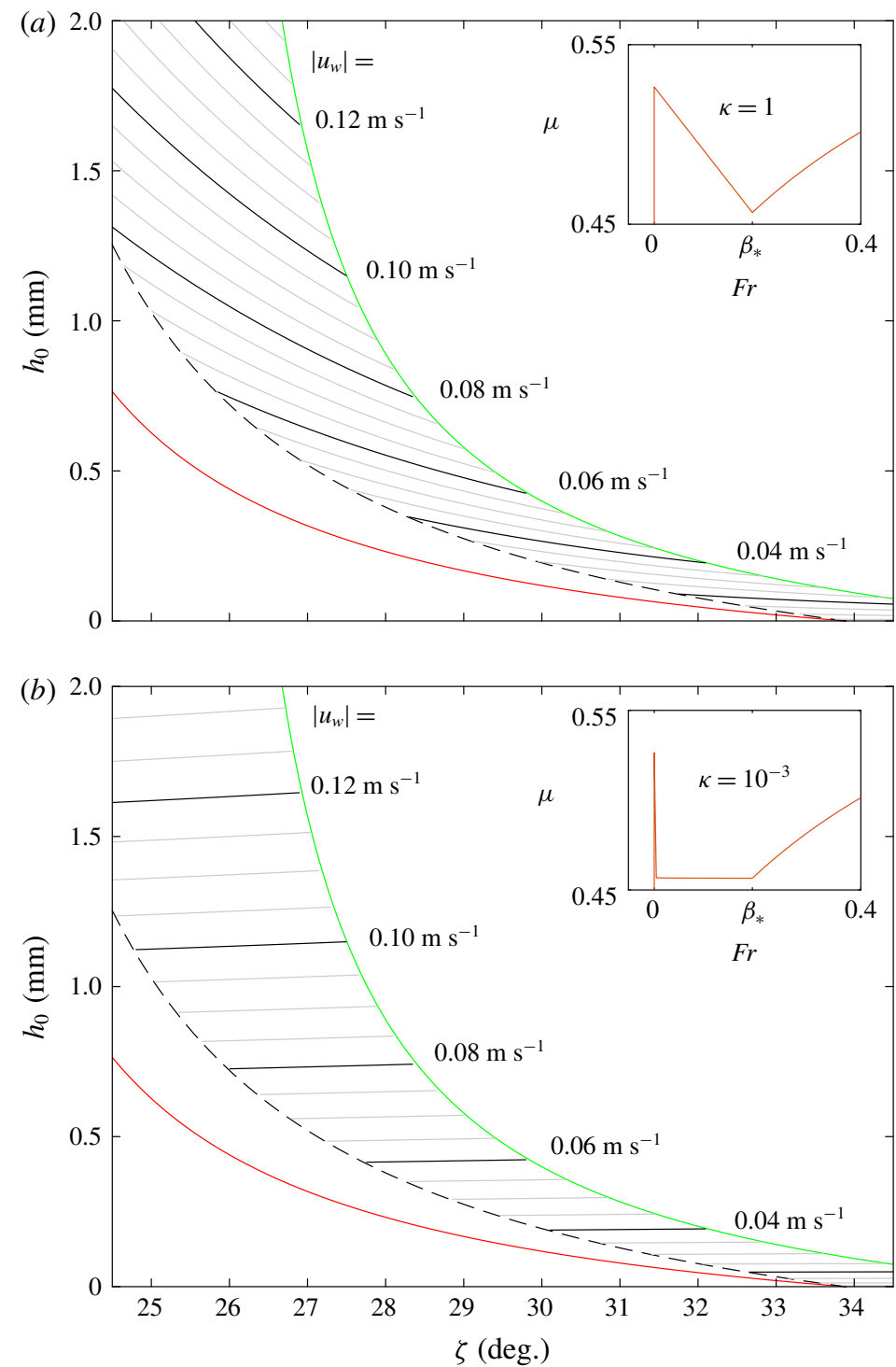

FIgURE 13. Contours of the retrogressive wave speed $\left|u_{w}\right|$ computed from (5.14) (black and grey solid lines), plotted with respect to the slope angle $\zeta$ and the initial layer thickness $h_{0}$. Solutions exist for $h_{0}$ between $h_{0}=h_{0}^{\min }(\zeta)$ (black dotted line) and $h_{0}=h_{0}^{\max }(\zeta)$ (green line). The inset shows the friction coefficient $\mu$ as a function of Froude number for $h=1 \mathrm{~mm}$. In (a) linear interpolation of the friction is used in the intermediate regime $(\kappa=1)$, leading to the experimentally observed increase in $\left|u_{w}\right|$ with increasing $\zeta$. In $(b)$ a nonlinear interpolation is used $\left(\kappa=10^{-3}\right.$, as suggested by Pouliquen \& Forterre 2002), leading to the opposite relationship between $\left|u_{w}\right|$ and $\zeta$. Note that in both of these plots the value of $\beta_{*}$ in table 1 is used.

the experiments in figure 12. While the scatter in experimental data make it difficult to infer the precise functional form of the friction coefficient in the intermediate regime, the clear increase in $\left|u_{w}\right|$ observed with increasing $\zeta$ is strong evidence that 
the transition to the static failure criterion at $F r=0$ is less rapid than that suggested by Pouliquen \& Forterre (2002). Note that the solution for the original friction law of Pouliquen \& Forterre (2002) in figure 12, with $\beta_{*}=\beta$, has a much wider region in which retrogressive failures form than observed in experiments.

When $\kappa=1$ the retrogressive wave speed (5.22) is an increasing function of the initial layer thickness $h_{0}$. For a given slope angle $\zeta$, the fastest and slowest retrogressive waves therefore occur at the boundaries $h_{0}=h_{0}^{\max }$ and $h_{0}=h_{0}^{\min }$ defined in (5.18) and (5.19), respectively. For the thickest initial static layer $h_{0}=h_{0}^{\max }$ the critical point $h_{c}$ takes its maximum value of $h_{\text {start }}$ and the velocity $\bar{u}_{c}$ is zero, as shown in figure 9. It follows, from (5.14) that the maximum upslope-propagating wave speed is

$$
\left|u_{w}\right|=\left|u_{w}\right|^{\max }=\sqrt{g h_{\text {start }} \cos \zeta} .
$$

Conversely, when $h_{0}=h_{0}^{\text {min }}$, the critical thickness $h_{c}$ takes its minimum value of $h_{*}$ and the Froude number $F r_{c}$ takes its maximum value of $\beta_{*}$ as shown in figure 9. This produces the slowest retrogressive wave speed for a given thickness $h_{0}$, which using (3.12) and (5.22), is

$$
\left|u_{w}\right|=\left|u_{w}\right|^{\min }=\left(1-\beta_{*}\right) \sqrt{g h_{*} \cos \zeta}=\left(1-\beta_{*}\right) \sqrt{\left(\beta_{*} / \beta\right) g h_{\text {stop }} \cos \zeta} .
$$

This is also the wave speed at the onset of retrogressive failure at $\zeta=\zeta^{\min }$.

\section{Conclusions and discussion}

Frictional hysteresis (Daerr \& Douady 1999; Daerr 2001; Aranson \& Tsimring 2002; Pouliquen \& Forterre 2002; Edwards et al. 2017, 2019) results in a range of thicknesses over which both static and moving layers of material can coexist in a granular flow. This property is fundamental to the retrogressive failures studied here, which combine a thicker static and thinner flowing layer. In more complex three-dimensional flows, hysteresis gives rise to a wide range of subtle morphological features on erodible beds, such as incised troughs (Daerr \& Douady 1999; Edwards et al. 2017), super-elevated channels and static levees (Edwards et al. 2017). It also plays a crucial role in the selection of the height and width of self-channelised flows on non-erodible beds (Rocha et al. 2019), which can significantly extend the run-out distance of hazardous geophysical mass flows (Félix \& Thomas 2004; Mangeney et al. 2007; Johnson et al. 2012; Kokelaar et al. 2014).

In this paper, it is shown experimentally that a static layer of thickness $h_{0}=h_{\text {stop }}\left(\zeta_{0}\right)$ on a chute inclined at angle $\zeta \in\left(\zeta_{0}, \zeta_{\text {start }}\right)$ can be eroded by a planar retrogressive failure, which separates static and flowing material and propagates upslope at constant speed (figures 1-5). These planar retrogressive failures occur when the deposit is perturbed along a straight line across the slope rather than just at a single point, as in the experiments of Daerr \& Douady (1999). The spatial uniformity in the cross-slope $y$ direction allows the whole process to be modelled with a relatively simple one-dimensional depth-averaged avalanche model that uses the non-monotonic effective basal friction law of Edwards et al. (2017, 2019).

Both direct numerical simulations (figures 7-8) and the experimental space-time plot (figure 4) suggest that the retrogressive failure rapidly develops into a travelling wave. In $\S 5$ the travelling wave ansatz is used to derive exact solutions for the speed of the wave $u_{w}$ and the associated initial deposit height $h_{0}$, which are dependent on a critical point where the flow has thickness $h_{c}$. This critical thickness is determined 
by the requirements that (i) it is a steady uniform flow solution (5.10) to the depthaveraged momentum balance (3.2) with the intermediate friction law (3.7) and (ii) the wave speed is equal to that of the upward hyperbolic characteristic. As a result, at the critical point, both the numerator and the denominator of the ODE (5.5) are zero and hence solutions exist that smoothly pass through $h=h_{c}$ with a finite gradient, connecting the static and flowing layers upstream and downstream of the wave.

The exact travelling wave solution accurately predicts the range of slope angles $\zeta$ and initial layer thicknesses $h_{0}$ for which retrogressive waves exist. The lower phase boundary, separating upslope propagating retrogressive failures from failures that only propagate downslope, is a sensitive test of the thinnest possible steady uniform flow. Figure 11 shows that this boundary is in excellent agreement with the experimentally determined phase boundary and is therefore supportive of Edwards et al.'s (2019) idea that the transition between intermediate and dynamic regimes occurs at constant Froude number $F r=\beta_{*}>\beta$, rather than at $F r=\beta$ (Pouliquen \& Forterre 2002). At this onset of retrogressive failure, a jump to a finite non-zero wave speed is correctly predicted by the model (figure 12), which is a prediction that has previously been obtained only through the introduction of an additional order parameter and associated governing equation (Aranson \& Tsimring 2001, 2002).

The wave speed (5.14) is dependent on the velocity and thickness at the critical point, which lies in the intermediate friction regime as shown in figure 9. Since steady uniform flows in this regime are inherently unstable and so are not observable experimentally, the retrogressive wave therefore provides a very important means of probing the functional form of the friction law in this region. The model captures the experimental trend that, for a fixed value of the initial layer $h_{0}$, waves move upslope faster at higher inclinations (figure 12). In contrast, the highly nonlinear interpolation suggested by Pouliquen \& Forterre (2002) predicts a slight decrease in the wave speed with increasing inclination angle, which is the opposite trend to that observed in the experiments. Our results therefore provide new evidence that the friction coefficient varies appreciably through the whole range $0<\mathrm{Fr}<\beta_{*}$ and that a linear interpolation with $\kappa=1$ is a more accurate parametrisation than using the value of $\kappa=10^{-3}$ of Pouliquen \& Forterre (2002).

The experiments and modelling of this paper indicate that for thin flows the apparent basal friction rises appreciably when the Froude number decreases below $\beta_{*}=0.19$. This suggests that the higher friction associated with static grains may play a role in more rapid flows (up to $F r \approx 0.19$ ) than has been suggested previously (Pouliquen \& Forterre 2002). A possible mechanism for this is that the failure of grains occurs through (rapid) progressive erosion, in which grains at the surface are the first to start flowing, followed by those near the bed. The increase in basal friction inferred for $\mathrm{Fr}<\beta_{*}$ would then be a consequence of depth integrating over a flow in which only the uppermost part is flowing. The steady-state velocity profiles predicted by discrete particle simulations and non-local rheological models of shallow flows (Silbert, Landry \& Grest 2003; Kamrin \& Henann 2015) are broadly supportive of this hypothesis, with the lower part of the velocity profile of slow, shallow flows exhibiting very little shear.

The retrogressive failure wave exemplifies several physical mechanisms that have yet to be fully incorporated into non-depth-integrated models of granular rheology. The flow depends crucially on non-local rheological behaviour, not only in flowing regions where the non-locality leads to the minimum flow thickness $h_{*}$, but also in static layers where the non-locality results in a maximum stable layer thickness $h_{\text {start }}$. Frictional hysteresis plays an equally important role, allowing these static and flowing 
layers to co-exist at the same slope angle. While many models of granular rheology are based on steady-state behaviour, the retrogressive wave is highly transient: the transition from static to flowing equilibrium states in the wave takes less than $100 \mathrm{~ms}$. Furthermore, the critical point analysis suggests that the speed of the retrogressive wave is sensitive to this transient flow within the wave itself, not simply on the steady states either side of it. The retrogressive wave may therefore provide a stringent test case for future rheological models that aim to capture non-local, hysteretic and transient phenomena in granular flows.

\section{Acknowledgements}

This research was supported by NERC grants NE/E003206/1 and NE/K003011/1 as well as EPSRC grants EP/I019189/1, EP/K00428X/1 and EP/M022447/1. J.M.N.T.G. is a Royal Society Wolfson Research Merit Award holder (WM150058) and an EPSRC Established Career Fellow (EP/M022447/1). F.M.R. acknowledges financial support from CNPq, Conselho Nacional de Desenvolvimento Científico e Tecnológico - Brazil. A.S.R. would like to thank C. Tregaskis and D. Tsuji for their help with performing the experiments. All research data supporting this publication are directly available within this publication.

\section{Supplementary movies}

Supplementary movies are available at https://doi.org/10.1017/jfm.2019.215.

\section{REFERENCES}

Aradian, A., Raphä̈L, É. \& De Gennes, P.-G. 2002 Surface flows of granular materials: a short introduction to some recent models. C. R. Phys. 3 (2), 187-196.

ARANSON, I. S. \& TSIMring, L. S. 2001 Continuum description of avalanches in granular media. Phys. Rev. E 64, 020301(R).

ARAnson, I. S. \& TSIMring, L. S. 2002 Continuum theory of partially fluidized granular flows. Phys. Rev. E 65, 061303.

BAKer, J. L., BARKer, T. \& Gray, J. M. N. T. $2016 a$ A two-dimensional depth-averaged mu(I)rheology for dense granular avalanches. J. Fluid Mech. 787, 367-395.

BALmForth, N. J. \& MCElwaine, J. N. 2018 From episodic avalanching to continuous flow in a granular drum. Granul. Matt. 20, 52.

Bouchaud, J. P. \& Cates, M. E. 1998 Triangular and uphill avalanches of a tilted sandpile. Granul. Matt. 1 (2), 101-103.

Bouchaud, J. P., Cates, M. E., Prakesh, J. R. \& Edwards, S. F. 1994 A model for the dynamics of sandpile surfaces. J. Phys. I France 4 (10), 1383-1410.

Bouchut, F. \& WestDickenberg, M. 2004 Gravity driven shallow water models for arbitrary topography. Commun. Math. Sci. 2 (3), 359-389.

Christen, M., Kowalski, J. \& BARTelt, P. 2010 RAMMS: numerical simulation of dense snow avalanches in three-dimensional terrain. Cold Reg. Sci. Technol. 63 (1-2), 1-14.

DAErr, A. 2001 Dynamical equilibrium of avalanches on a rough plane. Phys. Fluids 13 (7), $2115-2124$.

DAerr, A. \& Doundy, S. 1999 Two types of avalanche behaviour in granular media. Nature 399 (6733), 241-243.

Denlinger, R. P. \& IVERson, R. M. 2001 Flow of variably fluidized granular masses across three-dimensional terrain 2. Numerical predictions and experimental tests. J. Geophys. Res. 106 (B1), 553-566. 
Doyle, E. E., Hogg, A. J. \& MAder, H. M. 2011 A two-layer approach to modelling the transformation of dilute pyroclastic currents into dense pyroclastic flows. Proc. R. Soc. Lond. A 467 (2129), 1348-1371.

EDWARDS, A. N. \& GRAY, J. M. N. T. 2015 Erosion-deposition waves in shallow granular freesurface flows. J. Fluid Mech. 762, 35-67.

Edwards, A. N., Russell, A. S., Johnson, C. G. \& Gray, J. M. N. T. 2019 Frictional hysteresis and particle deposition in granular free-surface flows. J. Fluid Mech. (submitted).

Edwards, A. N., Viroulet, S., KokelaAR, B. P. \& Gray, J. M. N. T. 2017 Formation of levees, troughs and elevated channels by avalanches on erodible slopes. J. Fluid Mech. 823, $278-315$.

Eke, E., Viparelli, E. \& PARKer, G. 2011 Field-scale numerical modeling of breaching as a mechanism for generating continuous turbidity currents. Geosphere 7 (5), 1063-1076.

FÉlix, G. \& Thomas, N. 2004 Relation between dry granular flow regimes and morphology of deposits: formation of levées in pyroclastic deposits. Earth Planet. Sci. Lett. 221, 197-213.

Fischer, J. T., Kowalski, J. \& Pudasaini, S. P. 2012 Topographic curvature effects in applied avalanche modeling. Cold Reg. Sci. Technol. 74-75, 21-30.

GDR-MiDi 2004 On dense granular flows. Eur. Phys. J. E 14 (4), 341-365.

Glimsdal, S., L'Heureux, J. S., Harbitz, C. B. \& Lovholt, F. 2016 The 29th january 2014 submarine landslide at statland, norway-landslide dynamics, tsunami generation, and run-up. Landslides 13, 1435-1444.

Goujon, C., Thomas, N. \& Dalloz-Dubrujeaud, B. 2003 Monodisperse dry granular flows on inclined planes: role of roughness. Euro. Phys. J. E 11 (2), 147-157.

GRAY, J. M. N. T. \& EDWARDS, A. N. 2014 A depth-averaged $\mu(I)$-rheology for shallow granular free-surface flows. J. Fluid Mech. 755, 503-544.

Gray, J. M. N. T., TAI, Y. C. \& Noelle, S. 2003 Shock waves, dead-zones and particle-free regions in rapid granular free-surface flows. J. Fluid Mech. 491, 161-181.

Gray, J. M. N. T., Wieland, M. \& Hutter, K. 1999 Free surface flow of cohesionless granular avalanches over complex basal topography. Proc. R. Soc. Lond. A 455 (1985), 1841-1874.

Grigorian, S. S., Eglit, M. E. \& IAKIMOV, I. L. 1967 New statement and solution of the problem of the motion of snow avalanche. Snow, Avalanches \& Glaciers. Tr. Vysokogornogo Geofizich Inst 12, 104-113.

IVERSON, R. M. 1997 The physics of debris flows. Rev. Geophys. 35 (3), 245-296.

IVERson, R. M. \& DENLINGER, R. P. 2001 Flow of variably fluidized granular masses across three-dimensional terrain 1. Coulomb mixture theory. J. Geophys. Res. 106 (B1), 537-552.

IVERSON, R. M. \& OUYANG, C. 2015 Entrainment of bed material by earth-surface mass flows: review and reformulation of depth-integrated theory. Rev. Geophys. 53 (1), 27-58.

Jing, L., KwoK, C. Y., Leung, Y. F. \& Sobral, Y. D. 2016 Characterization of base roughness for granular chute flows. Phys. Rev. E 94, 052901.

Johnson, C. G., Kokelaar, B. P., Iverson, R. M., Logan, M., LaHusen, R. G. \& GRAY, J. M. N. T. 2012 Grain-size segregation and levee formation in geophysical mass flows. J. Geophys. Res. 117, F01032.

Jop, P., Forterre, Y. \& Pouliquen, O. 2006 A constitutive law for dense granular flows. Nature Publishing Group 441 (7094), 727-730.

Kamrin, K. \& Henann, D. L. 2015 Nonlocal modeling of granular flows down inclines. Soft Matt. 11 (1), 179-185.

Köhler, A., McElwaine, J. N., Sovilla, B., Ash, M. \& Brennan, P. 2016 The dynamics of surges in the 3 February 2015 avalanches in Vallee de la Sionne. J. Geophys. Res. 121 (11), 2192-2210.

Kokelaar, B. P., Graham, R. L., Gray, J. M. N. T. \& Vallance, J. W. 2014 Fine-grained linings of leveed channels facilitate runout of granular flows. Earth Planet. Sci. Lett. 385, 172-180.

Kuo, C. Y., Tai, Y. C., Bouchut, F., Mangeney, A., Pelanti, M., Chen, R. F. \& Chang, K. J. 2009 Simulation of Tsaoling landslide, Taiwan, based on Saint Venant equations over general topography. Engng Geol. 104 (3-4), 181-189. 
Kurganov, A. \& TADMOR, E. 2000 New high-resolution central schemes for nonlinear conservation laws and convection-diffusion equations. J. Comput. Phys. 160 (1), 241-282.

Lovholt, F., Pedersen, G., Harbitz, C. B., Glimsdal, S. \& Kim, J. 2015 On the characteristics of landslide tsunamis. Phil. Trans. R. Soc. Lond. A 373, 20140376.

Luca, I., Hutter, K., TAI, Y. C. \& KuO, C. Y. 2009 A hierarchy of avalanche models on arbitrary topography. Acta Mechanica 205 (1-4), 121-149.

Mangeney, A., Bouchut, F., Thomas, N., Vilotte, J. P. \& Bristeau, M. O. 2007 Numerical modeling of self-channeling granular flows and of their levee-channel deposits. J. Geophys. Res. 112, F02017.

Mangeney, A., Roche, O., Hungr, O., Mangold, N., Faccanoni, G. \& Lucas, A. 2010 Erosion and mobility in granular collapse over sloping beds. J. Geophys. Res. 115, F03040.

Mangeney-Castelnau, A., Vilotte, J. P., Bristeau, M. O., Perthame, B., Bouchut, F., Simeoni, C. \& Yerneni, S. 2003 Numerical modeling of avalanches based on Saint Venant equations using a kinetic scheme. J. Geophys. Res. 108 (B11), 2527-2544.

Mastbergen, D., van den Ham, G., Cartigny, M., Koelewijn, A., De Kleine, M., Clare, M., Hizzett, J., AzPiroz, M. \& Vellinga, A. 2016 Multiple flow slide experiment in the Westerschelde Estuary, The Netherlands. In Submarine Mass Movements and their Consequences, Advances in Natural and Technological Hazards Research, vol. 41, pp. 241-249. Springer.

Natim, M., NaAim-Bouvet, F., Faug, T. \& Bouchet, A. 2004 Dense snow avalanche modeling: flow, erosion, deposition and obstacle effects. Cold Reg. Sci. Tech. 39 (2-3), 193-204.

Pitman, E. B., Nichita, C. C., Patra, A., Bauer, A., Sheridan, M. \& Bursik, M. 2003 Computing granular avalanches and landslides. Phys. Fluids 15 (12), 3638-3646.

Pouliquen, O. 1999a Scaling laws in granular flows down rough inclined planes. Phys. Fluids 11 (3), 542-548.

Pouliquen, O. $1999 \mathrm{~b}$ On the shape of granular fronts down rough inclined planes. Phys. Fluids 11 (7), 1956-1958.

Pouliquen, O. \& Forterre, Y. 2002 Friction law for dense granular flows: application to the motion of a mass down a rough inclined plane. J. Fluid Mech. 453, 133-151.

Pudasaini, S. P. \& Hutter, K. 2003 Rapid shear flows of dry granular masses down curved and twisted channels. J. Fluid Mech. 495, 193-208.

Rocha, F. M., Johnson, C. G. \& Gray, J. M. N. T. 2019 Self-channelisation and levee formation in monodisperse granular flows. J. Fluid Mech. (submitted).

Saingier, G., Deboeuf, S. \& Lagrée, P.-Y. 2016 On the front shape of an inertial granular flow down a rough incline. Phys. Fluids 28 (5), 053302.

SAmpl, P. \& Zwinger, T. 2004 Avalanche simulation with samos. Ann. Glaciol. 38 (1), 393-398.

SAVAGE, S. B. \& HUtTer, K. 1989 The motion of a finite mass of granular material down a rough incline. J. Fluid Mech. 199, 177-215.

SAVAGe, S. B.\& HutTer, K. 1991 The dynamics of avalanches of granular materials from initiation to runout. Part i: analysis. Acta Mechanica 86 (1-4), 201-223.

Silbert, L. E., Ertas, D., Grest, G. S., Halsey, T. C., Levine, D. \& Plimpton, S. J. 2001 Granular flow down an inclined plane: Bagnold scaling and rheology. Phys. Rev. E 64, 051302.

Silbert, L. E., LANDry, J. W. \& Grest, G. S. 2003 Granular flow down a rough inclined plane: transition between thin and thick piles. Phys. Fluids 15 (1), 1-10.

VAn den Berg, J. H., VAn Gelder, A.\& Mastbergen, D. R. 2002 The importance of breaching as a mechanism of subaqueous slope failure in fine sand. Sedimentology 49 (1), 81-95.

VARnes, D. J. 1978 Slope movement types and processes. In Landslides Analysis and Control. Washington Transportation Research Board, Special Report 176 (ed. R. L. Schuster \& R. J. Krizek), chap. 2. National Academy of Sciences.

Viroulet, S., Baker, J.L., Edwards, A. N., Johnson, C. G., Gualtema, C., Clavel, P. \& GrAY, J.M.N.T. 2017 Multiple solutions for granular flow over a smooth two-dimensional bump. J. Fluid Mech. 815, 77-116.

Viroulet, S., Baker, J. L., Rocha, F. M., Johnson, C. G., Kokelaar, B. P. \& Gray, J. M. N. T. 2018 The kinematics of bidisperse granular roll waves. J. Fluid Mech. 848, 836-875. 
Wiederseiner, S., Andreini, N., Epely-Chauvin, G., Moser, G., Monnereau, M., Gray, J. M. N. T. \& ANCEY, C. 2011 Experimental investigation into segregating granular flows down chutes. Phys. Fluids 23, 013301.

Wieland, M., Gray, J. M. N. T. \& Hutter, K. 1999 Channelized free-surface flow of cohesionless granular avalanches in a chute with shallow lateral curvature. J. Fluid Mech. 392, 73-100. 